\title{
DECOMPOSING REPLICABLE FUNCTIONS
}

\section{J. MCKAY AND DAVID SEVILLA}

\begin{abstract}
We describe an algorithm to decompose rational functions from which we determine the poset of groups fixing these functions.
\end{abstract}

\section{Introduction to replicable functions}

We assume familiarity with the notation and contents of $[\mathbf{1}],[\mathbf{3}],[\mathbf{5}]$ and $[\mathbf{7}]$. Replicable functions are definable in terms of a generalized Hecke operator or by constraints on their coefficients, see [8]. Each such function $f$ is fixed by a group $G_{f}$ that is commensurable with the modular group, $\operatorname{PSL}(2, \mathbb{Z})$. There is a natural poset formed by these stabilizer groups, considered up to conjugation by $z \mapsto k z, k \in \mathbb{Z}^{>0}$.

Replicable functions have a $q$-series (Fourier series) expansion at $i \infty$ of the form

$$
f(q)=\frac{1}{q}+\sum_{k \geqslant 1} a_{k} q^{k}, \quad q=e^{2 \pi i z}, \Im(z)>0, \quad \forall k: \quad a_{k} \in \mathbb{Z} .
$$

Cummins proves in [4] that a finite series implies that $f(q)=1 / q+c q, c \in\{0,1,-1\}$ - the modular fictions, exp, cos, sin which we shall hereafter ignore.

Computations suggest that there are 616 other replicable functions of which 171 are monstrous moonshine functions, see [2]. There is no satisfactory proof of the completeness of this list although it is compatible with several independent computational checks.

The following remarkable result of Norton is fundamental, see [8], [4].

TheOREM 1. A replicable function is determined by its coefficients in the Norton basis, $\left\{a_{k}\right\}, k \in B=\{1,2,3,4,5,7,8,9,11,17,19,23\}$.

\section{Algorithms for functional decomposition and computation of relations}

\subsection{Functional decomposition}

We sketch the theory of univariate rational decomposition and an algorithm for the computation of the poset of replicable functions with respect to rational relations.

Definition. In $T=\mathbb{Q}(t) \backslash \mathbb{Q}$ we define the binary operation of composition as

$$
g(t) \circ h(t)=g(h(t))=g(h)(t) .
$$

$(T, \circ)$ is a semigroup with $t$ as neutral element.

The first author is supported by NSERC.

The second author is partially supported by Spanish Ministry of Science grant MTM2007-67088. Received 3 September 2007, revised 21 December 2007; published 9 June 2008.

2000 Mathematics Subject Classification 11F06, 68W30 (primary), 20D08, 20C34 (secondary).

(c) 2008, J. MCKay and David Sevilla 
If $f=g \circ h$, we call this a decomposition of $f$ and say that $g$ is a left component of $f$ and $h$ is a right component of $f$. A decomposition is trivial if $g$ or $h$ is a unit with respect to composition.

Decompositions $f=g_{1} \circ h_{1}=\left(g_{1} \circ u\right) \circ\left(u^{-1} \circ h_{2}\right)$ are called equivalent, where $u$ is invertible with respect to composition and $u^{-1}$ is its functional inverse.

Given a rational function $f \in T$, we call it indecomposable if it is not a unit and all its decompositions are trivial. A decomposition of $f \in \mathbb{Q}(t)$ of length $r$, $f=g_{1} \circ \cdots \circ g_{r}$, is called refined if each $g_{i}$ is indecomposable.

The units with respect to composition are linear fractional transformations. The decomposition problem is: given $f \in \mathbb{Q}(t)$, compute all the decompositions of $f$, i.e., find a representative $\left(h_{i}, g_{i}\right)$ for each class of decompositions with respect to the equivalence relation above. Solving this problem leads to the computation of all refined decompositions.

Definition. For a non-constant rational function $f(t)=f_{N}(t) / f_{D}(t)$ with $f_{N}, f_{D} \in$ $\mathbb{Q}[t]$ and $\operatorname{gcd}\left(f_{N}, f_{D}\right)=1$ we define the degree of $f$ as

$$
\operatorname{deg} f=\max \left\{\operatorname{deg} f_{N}, \operatorname{deg} f_{D}\right\} .
$$

We also define $\operatorname{deg} a=0$ for all non-zero $a \in \mathbb{Q}$.

Because the solution to the problem may not be unique, most decomposition algorithms have two steps: first, we compute candidates for the right components, then check for their associated left components.

Remark. Given $f, h \in \mathbb{Q}(t)$, we can efficiently test if there is a $g \in \mathbb{Q}(t)$ with $f=g \circ h$. It is necessary that $\operatorname{deg} h \operatorname{divides} \operatorname{deg} f$. We then solve the equations resulting from the $q$-expansion of $f-(g \circ h)$. This is fast as the equations are linear.

We introduce a useful notion that will be the starting point for the decomposition algorithm, see $[\mathbf{6}]$ and $[\mathbf{1 0}]$.

Definition. Let $f=f_{N} / f_{D} \in \mathbb{Q}(t)$ with $f_{N}, f_{D} \in \mathbb{Q}[t], \operatorname{gcd}\left(f_{N}, f_{D}\right)=1$. We say that $f$ is in normal form when $\operatorname{deg} f_{N}>\operatorname{deg} f_{D}$ and $f_{N}(0)=0$ (or simply, $f(\infty)=\infty, f(0)=0)$.

\section{THEOREM 2. Let $f \in T$.}

(i) There exist units $u, v \in \mathbb{Q}(t)$ such that $u \circ f \circ v$ is in normal form, with both numerator and denominator monic.

(ii) Let $f \in \mathbb{Q}(t)$ be in normal form. If $f=g \circ h$, there is a unit $u$ such that $g \circ u$ and $u^{-1} \circ h$ are in normal form.

The following is the key to the decomposition algorithm.

Theorem 3. Let $f, g, h \in \mathbb{Q}(t)$ with $f=f_{N} / f_{D}, h=h_{N} / h_{D}$ where $f_{N}, f_{D}, h_{N}, h_{D} \in$ $\mathbb{Q}[t], \operatorname{gcd}\left(f_{N}, f_{D}\right)=1$ and $\operatorname{gcd}\left(h_{N}, h_{D}\right)=1$, and $f=g \circ h$. If $f, g, h$ are in normal form, then $h_{N} \mid f_{N}$ and $h_{D} \mid f_{D}$. 
Proof. Let

$$
g=\frac{t^{r}+c_{r-1} t^{r-1}+\cdots+c_{1} t}{d_{r-1} t^{r-1}+\cdots+d_{0}}, \quad d_{0} \neq 0
$$

then

$$
f=\frac{h_{N}^{r}+c_{r-1} h_{N}^{r-1} h_{D}+\cdots+c_{1} h_{N} h_{D}^{r-1}}{d_{r-1} h_{N}^{r-1} h_{D}+\cdots+d_{0} h_{D}^{r}}
$$

and, as the degree is multiplicative with respect to composition, there is no simplification in this expression. The result follows.

We describe the algorithm now.

Algorithm (Rational decomposition).

Input: $f \in T$.

Output: all non-trivial decompositions $(g, h)$ of $f$, if any exists.

A Compute $u$ and $v$ so that $\bar{f}=u \circ f \circ v$ is in normal form. Let $f_{N}, f_{D}$ be the monic numerator and denominator of $\bar{f}$.

B Factor $f_{N}$ and $f_{D}$. From this compute $D=\left\{\left(A_{1}, B_{1}\right), \ldots,\left(A_{m}, B_{m}\right)\right\}$, the set of pairs $(A, B)$ such that $A, B$ are monic polynomials dividing $f_{N}, f_{D}$ respectively. Set $i=1$.

C Check if there exists $g \in \mathbb{Q}(t)$ with $\bar{f}=g\left(A_{i} / B_{i}\right)$; if it does, add $\left(u^{-1}(g), h\left(v^{-1}\right)\right)$ to the list of decompositions of $f$.

D If $i<m$, increase $i$ and go to $\mathbf{C}$, otherwise return the list of decompositions.

AnALYSIS. The description above shows that the algorithm correctly computes at least one representative for each equivalence class of decompositions (an extra step would be needed to avoid having more than one representative for each decomposition class). The algorithm has exponential complexity due to the possibility of having an exponential number of candidates in the worst case. In practice, degree conditions reduce the number of candidates. In tests we have found that about $85 \%$ of the time is spent on the factoring, and the number of candidates is small (random polynomials are irreducible). Because of this, the algorithm is fast.

\subsection{Rational relations}

To find relations between two $q$-series we follow a simple procedure. We use the fact that replicable functions correspond to groups acting on the upper half plane, and a rational function of degree $n$ is an $n: 1$ map.

Algorithm (Computation of rational relations).

Input: two $q$-series $s_{1}, s_{2}$ as described in the introduction.

Output: all rational relations of the form $s_{1}\left(q^{k}\right)=f\left(s_{2}(q)\right), k \geqslant 1$.

A Compute the orders $e_{1}, \ldots, e_{r}$ of the generators $M_{1}, \ldots, M_{r}$ of the fundamental region of $s_{1}$. The hyperbolic area of the region is $A_{1}:=(r-2) \pi-\sum \pi / e_{i}$. Compute the area $A_{2}$ for $s_{2}$.

B If $d:=A_{2} / A_{1}$ is not an integer, then there are no relations. Otherwise, put $r=1$. 
C Let

$$
f=\frac{t^{d}+a_{d-1} t^{d-1}+\cdots+a_{0}}{t^{d-r}+b_{d-r-1} t^{d-r-1}+\cdots+b_{0}}
$$

and solve for $a_{i}, b_{j}$ the linear system given by $f\left(s_{2}\left(q^{r}\right)\right)-s_{1}(q)$.

D If there is a non-trivial solution to the system, store the corresponding $f$ and $r$. If $r<d$, increase $r$ and go to $\mathbf{C}$, otherwise return all relations found.

ANALYSIS. In each relation, the degree of the numerator is the ratio of the areas, and the difference between the degrees of numerator and denominator is the exponent of $q$ in the function $s_{2}$. In step $\mathbf{A}$, the orders of the non-identity elements are determined by the trace squared divided by the determinant. In step $\mathbf{C}$, solving for the $a_{i}, b_{j}$ requires less than $2 d$ coefficients.

REMARK. The values $k \geqslant 1$ correspond to the conjugation $z \mapsto k z, k \in \mathbb{Z}^{>0}$, that is, $q \mapsto q^{k}$.

\section{The computation}

For each of the 616 replicable functions, we have:

- the coefficients $a_{1}, \ldots, a_{23}$,

- parabolic and elliptic generators for the fixing groups, i.e. generators of the stabilizers of the vertices of a fundamental region.

For each pair of series we determine whether there is a rational relation between them as in Section 3.2. We decompose any rational relations as described in Section 3.1 in order to refine the decompositions, we repeat until we have all refined decompositions. In terms of the poset graph we use:

Algorithm (Poset refinement).

A Draw a vertex for each of the 616 functions.

B For each pair of functions $s_{1}$ and $s_{2}$, compute all rational relations of the form $s_{1}\left(q^{k}\right)=f\left(s_{2}(q)\right)$, if any. For each relation, draw a labelled directed edge

$$
s_{1} \stackrel{\operatorname{deg} f, k}{\longrightarrow} s_{2} \quad \text { where } s_{1}\left(q^{k}\right)=f\left(s_{2}(q)\right) .
$$

C For each of these, compute all the decompositions of $f$. For each decomposition $(g, h)$ compute $s_{3}\left(q^{j}\right):=h\left(s_{2}(q)\right), j \geqslant 1$ and replace the edge with the two edges

$$
s_{1} \stackrel{\operatorname{deg} g, k / j}{\longrightarrow} s_{3} \stackrel{\operatorname{deg} h, j}{\longrightarrow} s_{2}
$$

D Repeat step B until all the rational functions are indecomposable.

The computations described in this section were performed in a Pentium-IV $2 \mathrm{GHz}$ using Maple 7 . First, once the areas were known, it was seen that the maximum possible degree of a rational relation would be 96 . The search for rational relations took about 20 hours, about $10 \%$ of this time was spent in precomputing 200 coefficients for each series from the initial 23. We found 2419 relations in this 
step, we show their degrees below.

$\begin{array}{cccc}\text { degree } & \text { number } & \text { degree } & \text { number } \\ 2 & 698 & 16 & 52 \\ 3 & 243 & 18 & 60 \\ 4 & 422 & 20 & 2 \\ 5 & 26 & 24 & 71 \\ 6 & 333 & 28 & 2 \\ 8 & 178 & 30 & 8 \\ 9 & 40 & 32 & 4 \\ 10 & 14 & 36 & 40 \\ 12 & 209 & 48 & 5 \\ 14 & 4 & 72 & 2 \\ 15 & 6 & & \end{array}$

In Step C, we decompose all the functions we found previously, remove the repeated relations, and continue until all functions are indecomposable. In this way, and since our decomposition algorithm outputs all possible decompositions up to units, we ensure that we find all missing functions, if any, from the lists available. The computation of all possible decompositions is fundamental since there exists no formal proof of the completeness of our initial data. Our computation provides this. The decomposition of all rational relations took around 30 hours overall. In the end, we obtained 1049 indecomposable rational relations. We summarize them in Table 1 in the appendix. We also list the connected components of the graph there.

For each function we give its immediate predecessors and successors. For each edge we give two numbers, the degrees of the numerator and denominator of the rational relation; the first is the degree of the relation, and the power of $q$ is given by the difference of the two numbers. For example, $(1 A, 2: 0)$ in line $2 a$ means that $j\left(q^{2}\right)$ is a degree two polynomial in the principal modulus $2 a$. Notice that in some cases there are two edges between two given functions.

\section{Remarks}

It is noteworthy that for two series $s_{1}, s_{2}$ we may find more than one relation, i.e. $s_{1}\left(q^{k_{1}}\right)=f_{1}\left(s_{2}(q)\right)$ and $s_{1}\left(q^{k_{2}}\right)=f_{2}\left(s_{2}(q)\right)$ with $k_{1} \neq k_{2}$. By computation of a resultant, we can find a polynomial relation of the type $P\left(s_{1}(q), s_{1}\left(q^{k_{1} / k_{2}}\right)\right)=0$.

Computation reveals a remarkable fact about the relation between $j$ (labelled $f=1 A)$ and the principal modulus for $\Gamma(3), t=s(z / 3)$ where $s=\left(\eta(q) / \eta\left(q^{9}\right)\right)^{3}+3$, labelled $9 B$. Specifically, refined decomposition chains of different lengths exist for

$$
\begin{aligned}
& f=\frac{t^{3}\left(t^{3}+6^{3}\right)^{3}}{\left(t^{3}-3^{3}\right)^{3}} \\
& \text { namely } f=t^{3} \circ \frac{t(t-12)}{t-3} \circ \frac{t(t+6)}{t-3} \\
& \text { and } f=\frac{t^{3}(t+24)}{t-3} \circ \frac{t\left(t^{2}-6 t+36\right)}{t^{2}+3 t+9} .
\end{aligned}
$$

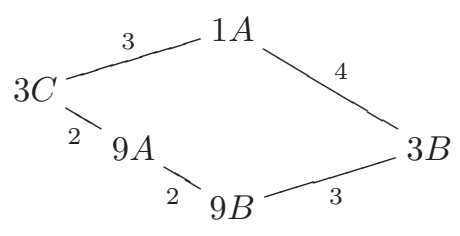


We believe this is the first example of a rational function in $\mathbb{Q}(t)$ with refined decomposition chains of different lengths. This does not occur with polynomials, see $[\mathbf{9}]$.

Norton points out that to every component (other than the fictions) there is at least one function that is either monstrous or the translate of a monstrous function.

\section{Appendix}

Table 1: Table of minimal relations sorted by middle function

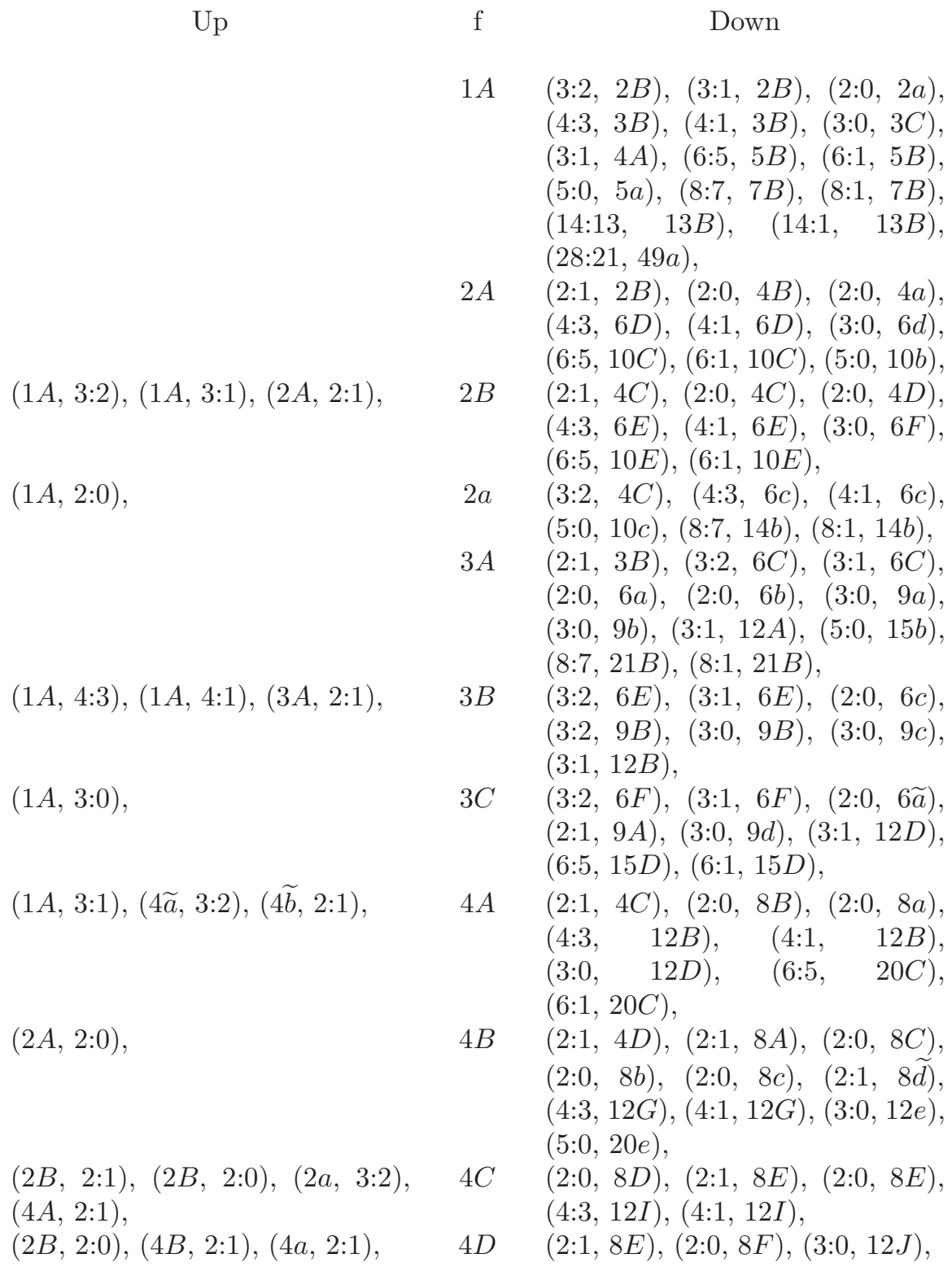


$\mathrm{Up}$

(2A, 2:0),

(1A, 6:5), (1A, 6:1), (5A, 2:1),

$(1 A, 5: 0)$,

$(6 A, 2: 1)$,

$(3 A, 3: 2),(3 A, 3: 1),(6 A, 2: 1)$,

(2A, 4:3), (2A, 4:1), (6A, 2:1),

$(2 B, 4: 3),(2 B, 4: 1),(3 B, 3: 2)$, (3B, 3:1), $(6 B, 2: 1),(6 C, 2: 1)$, $(6 D, 2: 1)$, $(2 B, 3: 0),(3 C, 3: 2),(3 C, 3: 1)$, $(6 d, 2: 1)$, (3A, 2:0), (3A, 2:0),

$(2 a, 4: 3),(2 a, 4: 1),(3 B, 2: 0)$ $(6 a, 2: 1),(6 b, 2: 1)$,

(2A, 3:0),

$(3 C, 2: 0)$, $\mathrm{f}$

$4 a$

$4 \widetilde{a}$

$$
\widetilde{4 b}
$$

$5 A$

$5 B$

$5 a$

$6 A \quad(2: 1,6 B),(2: 1,6 C),(2: 1,6 D)$,

$6 B$

$6 C$

$6 D$ $(2: 0,12 C),(2: 0,12 a),(2: 0,12 b)$, $(3: 0,18 a),(3: 0,18 h),(5: 0,30 e)$, $(2: 1,6 E),(2: 0,12 F),(3: 0,18 d)$, $(2: 1,6 E),(2: 1,12 E),(2: 0,12 E)$, $(2: 0,12 c),(2: 0,12 d),(2: 1,12 \widetilde{h})$, $(3: 0,18 e)$,

$(2: 1,6 E),(2: 0,12 G),(3: 2,18 A)$, $(3: 0,18 A)$,

$(2: 1,12 I),(2: 0,12 I),(3: 2,18 D)$, $(3: 0,18 D)$,

$6 F \quad(2: 0,12 J),(2: 0,12 \widetilde{j}),(2: 1,18 C)$, $(2: 1,36 \widetilde{m})$,

$6 a \quad(2: 1,6 c),(3: 2,12 E),(3: 0,18 f)$,

$6 b \quad(2: 1,6 c),(3: 2,12 H),(3: 2,12 c)$, $(3: 2,12 \widetilde{h}),(3: 0,18 b),(3: 0,18 g)$, $(5: 0,30 f)$,

$6 c \quad(3: 2,12 I),(3: 0,18 i)$,

$6 d \quad(2: 1,6 F),(2: 0,12 e),(2: 0,12 f)$, $(2: 0,12 \widetilde{i}),(2: 1,18 B),(3: 0,18 j)$, $(2: 1,18 \widetilde{a})$,

$6 \widetilde{a} \quad(3: 2,12 \widetilde{j}),(3: 2,36 \widetilde{l}),(3: 2,36 \widetilde{m})$, $7 A \quad(2: 1,7 B),(3: 2,14 B),(3: 1,14 B)$, $(2: 0,14 a),(2: 0,14 c),(3: 0,21 C)$, $(3: 1,28 B),(5: 0,35 a)$, 


\section{Up}

(1A, 8:7), (1A, 8:1), (7A, 2:1), $(4 B, 2: 1)$,

$(4 A, 2: 0),(8 \widetilde{b}, 2: 1),(8 \widetilde{c}, 2: 1)$,

$(4 B, 2: 0)$,

(4C, $2: 0),(8 B, 2: 1),(8 a, 2: 1)$, (4C, 2:1), (4C, 2:0), (4D, 2:1), $(8 A, 2: 1),(8 \widetilde{d}, 2: 1)$,

(4D, 2:0), (8b, 2:1), (8c, 2:1), $(4 A, 2: 0),(8 \widetilde{a}, 3: 2)$,

$(4 B, 2: 0)$,

$(4 B, 2: 0)$,

$(4 \widetilde{a}, 2: 0)$,

$(4 \widetilde{b}, 2: 0)$,

$(4 \widetilde{b}, 2: 0)$,

$(4 B, 2: 1)$,

$(3 C, 2: 1)$,

$(3 B, 3: 2),(3 B, 3: 0),(9 A, 2: 1)$,

(3A, 3:0),

(3A, 3:0),

$(3 B, 3: 0),(9 b, 2: 1)$,

$(3 C, 3: 0)$,

f

$7 B$

$8 A$

$8 B$

$8 C$

$8 D$

$8 E$

$8 F$

$8 a$

$8 b$

$8 c$

$8 \widetilde{a}$

$8 \widetilde{b}$

$8 \widetilde{c}$

$8 \widetilde{d}$

$9 A$

$9 B$

$9 a$

$9 b$

$9 c$

$9 d$

$10 A$

$(5 A, 3: 2),(5 A, 3: 1),(10 A, 2: 1), \quad 10 B$

(2A, 6:5), (2A, 6:1), (10A, 2:1), $(10 A, 2: 1)$,
Down

$(2: 0,14 b)$,

$(2: 1,8 E),(2: 0,16 A),(4: 3,24 C)$, $(4: 1,24 C)$,

$(2: 1,8 D),(2: 1,16 C),(2: 0,16 a)$, $(2: 0,16 b),(2: 0,16 c),(2: 1,16 \widetilde{e})$, $(3: 0,24 E)$,

$(2: 1,16 A),(2: 0,16 e),(2: 0,16 f)$, $(2: 1,16 \widetilde{d}),(4: 3,24 G),(4: 1,24 G)$, $(5: 0,40 e)$,

$(2: 1,16 B),(2: 0,16 d)$,

$(2: 1,16 B),(2: 0,16 B)$,

$(3: 0,24 J)$,

$(2: 1,8 D),(4: 3,24 c),(4: 1,24 c)$, $(2: 1,8 F),(2: 1,16 A),(2: 0,16 g)$, $(2: 0,16 h),(3: 0,24 i)$,

$(2: 1,8 F),(2: 1,16 \widetilde{d}),(3: 0,24 j)$, $(3: 2,8 a),(4: 3,24 \widetilde{c}),(4: 1,24 \widetilde{c})$, $(5: 0,40 \widetilde{g}),(8: 7,56 \widetilde{b}),(8: 1,56 \widetilde{b})$, $(2: 1,8 B),(2: 0,16 \widetilde{a}),(4: 3,24 \widetilde{h})$, $(4: 1,24 \widetilde{h}),(3: 0,24 \widetilde{l}),(5: 0,40 \widetilde{j})$, $(2: 1,8 B),(2: 0,16 \widetilde{b}),(2: 0,16 \widetilde{c})$, $(3: 0,24 \widetilde{n}),(6: 5,40 \widetilde{e}),(6: 1,40 \widetilde{e})$, $(2: 1,8 E),(2: 0,16 \widetilde{d}),(4: 3,24 \widetilde{o})$, $(4: 1,24 \widetilde{o})$,

$(2: 1,9 B),(3: 2,18 C),(3: 1,18 C)$, $(2: 0,18 c),(3: 0,27 a),(3: 0,27 b)$, $(3: 1,36 A)$,

$(3: 2,18 D),(3: 1,18 D),(3: 0,27 c)$, $(3: 1,36 B)$,

$(2: 0,18 b),(3: 2,18 e),(3: 1,18 e)$, $(3: 2,27 A),(3: 1,36 b),(5: 0,45 c)$, $(2: 1,9 c),(2: 0,18 f),(2: 0,18 g)$, $(3: 2,27 A),(3: 0,27 d),(3: 0,27 e)$, $(2: 0,18 i)$,

$(2: 1,27 b)$,

$(2: 1, \quad 10 B), \quad(2: 1, \quad 10 C)$, $(2: 1,10 D),(2: 0,20 B),(2: 0,20 a)$, $(2: 0,20 b),(3: 0,30 b)$,

$(2: 1,10 E),(2: 0,20 D),(2: 0,20 d)$, $(2: 1,20 \widetilde{g})$,

$10 C \quad(2: 1,10 E),(2: 0,20 c)$,

$10 D \quad(2: 1, \quad 10 E), \quad(2: 0, \quad 20 E)$, $(3: 0,30 E)$, 
Up

$(2 B, 6: 5),(2 B, 6: 1),(5 B, 3: 2)$, $(5 B, 3: 1),(10 B, 2: 1),(10 C, 2: 1)$, $(10 D, 2: 1)$,

(5A, 2:0),

(2A, 5:0),

$(2 a, 5: 0),(5 a, 2: 0)$,

$(3 A, 3: 1),(12 \widetilde{a}, 3: 2),(12 \widetilde{d}, 2: 1)$,

(3B, 3:1), (4A, 4:3), (4A, 4:1), $(12 A, 2: 1),(12 \widetilde{b}, 3: 2),(12 \widetilde{e}, 2: 1)$, $(12 \widetilde{f}, 2: 1)$, $(6 A, 2: 0)$,

$(3 C, 3: 1),(4 A, 3: 0),(12 \widetilde{c}, 3: 2)$, $(12 \widetilde{g}, 2: 1)$,

$(6 C, 2: 1),(6 C, 2: 0),(6 a, 3: 2)$, $(12 A, 2: 1)$, $(6 B, 2: 0),(12 C, 2: 1),(12 b, 2: 1)$,

(4B, 4:3), (4B, 4:1), (6D, 2:0), $(12 C, 2: 1),(12 a, 2: 1)$,

$(6 b, 3: 2),(12 A, 2: 1)$,

$(4 C, 4: 3),(4 C, 4: 1),(6 E, 2: 1)$, $(6 E, 2: 0),(6 c, 3: 2),(12 B, 2: 1)$, (12E, 2:1), (12H, 2:1), (12c, 2:1), $(12 \widetilde{h}, 2: 1)$,

$(4 D, 3: 0),(6 F, 2: 0),(12 e, 2: 1)$, (12f, 2:1),

(6A, 2:0),

$(6 A, 2: 0)$,

(6C, 2:0), (6b, 3:2), (6C, 2:0), (12a, 2:1), (12b, 2:1), $(4 B, 3: 0),(6 d, 2: 0)$,

$(4 a, 3: 0),(6 d, 2: 0)$, $\mathrm{f}$

Down

$10 E$

$10 a$

$(3: 2,20 F),(3: 2,20 d),(3: 2,20 \widetilde{g})$, $(4: 3,30 c),(4: 1,30 c)$,

$10 b \quad(2: 0,20 e),(3: 2,50 A)$,

$10 c$

$11 A(3: 2,22 B),(3: 1,22 B),(2: 0,22 a)$, $(4: 3,33 A),(4: 1,33 A),(3: 1,44 A)$,

$12 A \quad(2: 1, \quad 12 B), \quad(2: 1, \quad 12 E)$, $(2: 1,12 H),(2: 0,24 A),(2: 0,24 a)$, $(2: 0,24 b),(3: 0,36 b)$,

$12 B \quad(2: 1,12 I),(2: 0,24 c),(3: 2,36 B)$, $(3: 0,36 B)$,

$12 C \quad(2: 1, \quad 12 F), \quad(2: 1, \quad 12 G)$, $(2: 1,24 B),(2: 0,24 d),(2: 0,24 e)$, $(2: 0,24 f),(2: 0,24 g),(2: 1,24 \widetilde{j})$, $(2: 1,24 \widetilde{k}),(2: 1,24 \widetilde{m}),(3: 0,36 d)$, $12 D \quad(2: 0,24 E),(2: 0,24 \widetilde{s}),(2: 1,36 A)$, $(2: 1,36 \widetilde{l})$,

$(2: 1,12 I),(2: 0,24 D),(2: 0,24 h)$, $(2: 1,24 \widetilde{q})$,

$12 F \quad(2: 0,24 F),(2: 1,24 I),(2: 1,24 \widetilde{r})$, $(3: 0,36 g)$,

$12 G \quad(2: 1,24 C),(2: 0,24 G),(2: 1,24 \widetilde{o})$,

$12 H \quad(2: 1,12 I),(2: 0,24 H)$, $12 I$

$12 J \quad(2: 0,24 J),(2: 1,72 \widetilde{o})$,

$12 a \quad(2: 1,12 G),(2: 1,12 d),(3: 0,36 h)$, $(5: 0,60 d)$,

$12 b \quad(2: 1,12 F),(2: 1,12 d),(3: 0,36 a)$, $(3: 0,36 c)$,

$12 c \quad(2: 1,12 I),(2: 1,24 h)$, $12 d \quad(2: 1,24 h),(2: 1,24 \widetilde{q}),(3: 0,36 e)$, $12 e \quad(2: 1,12 J),(2: 0,24 i),(2: 0,24 j)$, $(2: 0,24 \widetilde{t}),(2: 1,36 C),(2: 1,72 \widetilde{j})$, $(2: 1,72 \widetilde{k})$,

$12 f \quad(2: 1,12 J),(3: 0,36 i),(2: 1,36 \widetilde{o})$, 
$\mathrm{Up}$

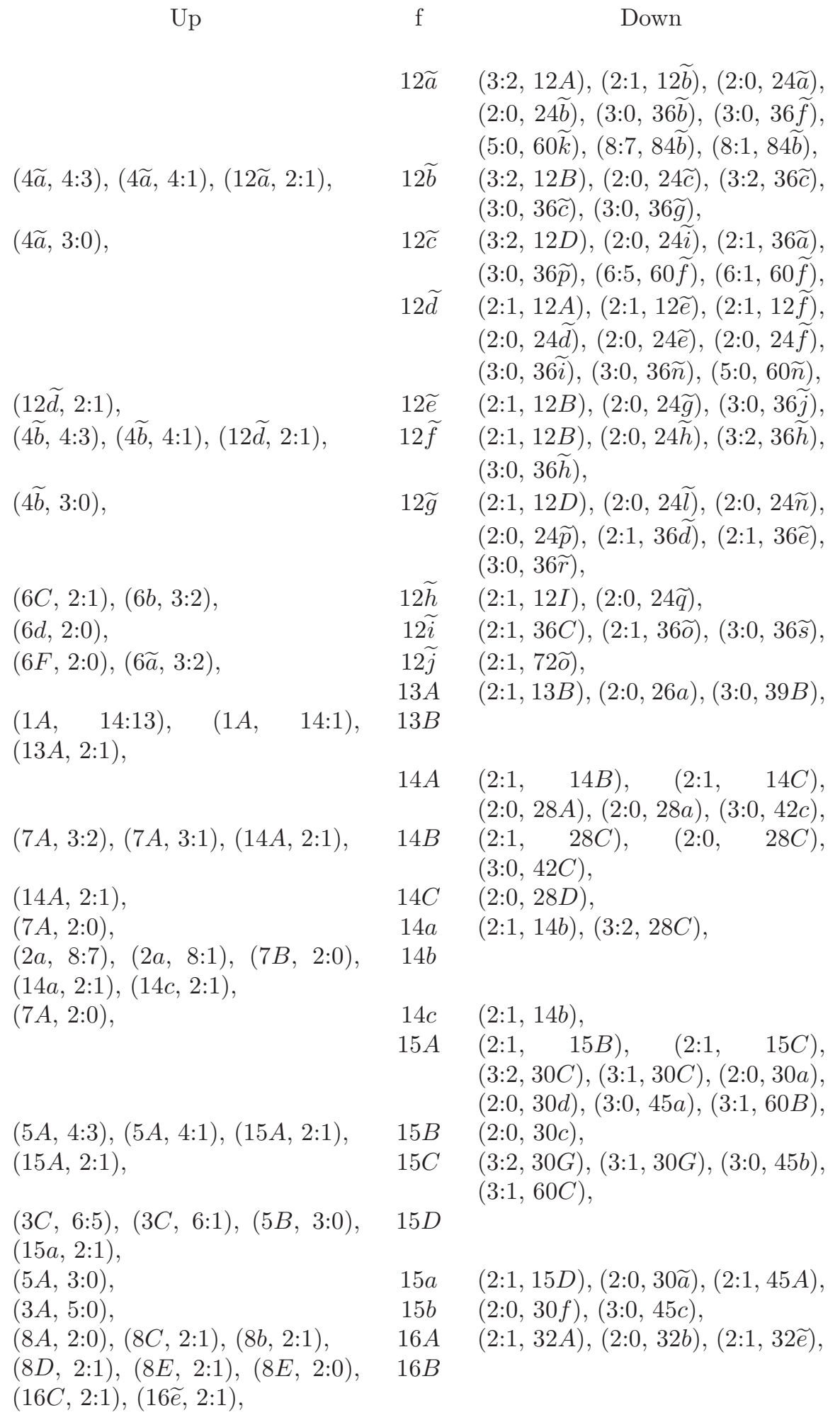


Up

$(8 B, 2: 1)$,

$(8 B, 2: 0),(16 \widetilde{b}, 2: 1),(16 \widetilde{c}, 2: 1)$,

$(8 B, 2: 0)$,

$(8 B, 2: 0)$,

$(8 D, 2: 0),(16 b, 2: 1),(16 c, 2: 1)$,

$(8 C, 2: 0)$,

$(8 C, 2: 0)$,

$(8 b, 2: 0)$,

$(8 b, 2: 0)$,

$(8 \widetilde{b}, 2: 0)$,

$(8 \widetilde{c}, 2: 0)$,

$(8 \widetilde{c}, 2: 0)$,

$(8 C, 2: 1),(8 c, 2: 1),(8 \widetilde{d}, 2: 0)$,

$(8 B, 2: 1)$,

$(6 D, 3: 2),(6 D, 3: 0),(18 B, 2: 1)$, $(6 d, 2: 1)$,

$(6 F, 2: 1),(9 A, 3: 2),(9 A, 3: 1), \quad 18 C$ $(18 B, 2: 1),(18 \widetilde{a}, 2: 1)$,

$(6 E, 3: 2),(6 E, 3: 0),(9 B, 3: 2), \quad 18 D$

$(9 B, 3: 1),(18 A, 2: 1),(18 C, 2: 1)$,

$(18 E, 2: 1)$,

$(18 B, 2: 1)$,

(6A, 3:0),

$(6 b, 3: 0),(9 a, 2: 0)$,

(9A, 2:0),

(6B, 3:0), (18a, 2:1),

$(6 C, 3: 0),(9 a, 3: 2),(9 a, 3: 1)$,

$(18 h, 2: 1)$,

(6a, 3:0), (9b, 2:0),

$(6 b, 3: 0),(9 b, 2: 0)$,

(6A, 3:0),

$(6 c, 3: 0),(9 c, 2: 0),(18 f, 2: 1), \quad 18 i$

$(18 g, 2: 1)$,

$(6 d, 3: 0)$,

$(6 d, 2: 1)$,

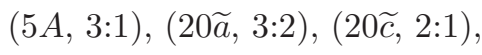

$16 C \quad(2: 1,16 B),(2: 0,32 B)$,

$16 a \quad(2: 1,32 B),(2: 0,32 a),(2: 1,32 \widetilde{d})$, $(3: 0,48 g)$,

$16 b \quad(2: 1,16 d),(2: 1,32 B),(2: 0,32 c)$, $(2: 0,32 d)$,

$16 c \quad(2: 1,16 d),(2: 1,32 \widetilde{d})$,

$16 d \quad(2: 0,32 e)$,

$16 e \quad(2: 1,32 \widetilde{c})$,

$16 f \quad(2: 1,32 \widetilde{c})$,

$16 g \quad(2: 1,32 b)$,

$16 h \quad(2: 1,32 b)$,

$16 \widetilde{a} \quad(4: 3,48 \widetilde{c}),(4: 1,48 \widetilde{c}),(5: 0,80 \widetilde{f})$,

$16 \widetilde{b}(2: 1,16 a),(2: 0,32 \widetilde{a}),(3: 0,48 \widetilde{l})$,

$16 \widetilde{c} \quad(2: 1,16 a),(2: 0,32 \widetilde{b}),(3: 0,48 \widetilde{m})$,

$16 \widetilde{d}(2: 0,32 \widetilde{c})$,

$16 \widetilde{e}(2: 1,16 B),(2: 0,32 \widetilde{d})$,

$17 A \quad(2: 0,34 a)$,

$18 A \quad(2: 1,18 D)$,

$18 B \quad(2: 1, \quad 18 A), \quad(2: 1, \quad 18 C)$, $(2: 1,18 E),(2: 0,36 C),(3: 0,54 a)$, $(2: 1,18 D),(2: 0,36 f),(2: 1,36 \widetilde{q})$,

$18 E \quad(2: 1,18 D),(3: 0,54 b)$,

$18 a \quad(2: 1,18 d),(2: 0,36 a),(2: 0,36 d)$, $(3: 2,54 A)$,

$18 b$

$18 c \quad(3: 2,36 D),(3: 2,36 f),(3: 2,36 \widetilde{q})$, $(3: 0,54 c)$,

$18 d \quad(2: 0,36 g)$,

$18 e \quad(2: 0,36 e)$,

$18 f \quad(2: 1,18 i)$,

$18 \mathrm{~g} \quad(2: 1,18 i),(3: 0,54 d)$,

$18 h \quad(2: 1,18 e),(2: 0,36 c),(2: 0,36 h)$, $(3: 2,54 A)$,

$18 j \quad(2: 0,36 i),(2: 0,36 \widetilde{s}),(2: 1,54 \widetilde{a})$, $18 \widetilde{a} \quad(2: 1,18 C),(2: 0,36 \widetilde{o}),(3: 0,54 \widetilde{a})$, $19 A \quad(2: 0,38 a),(3: 0,57 A)$,

$20 A \quad(2: 1, \quad 20 C), \quad(2: 1, \quad 20 F)$, $(2: 0,40 B),(2: 0,40 a)$, 
Up

$(10 A, 2: 0)$,

(4A, 6:5), (4A, 6:1), (5B, 3:1),

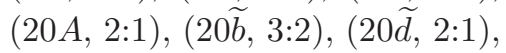
$(20 \widetilde{e}, 2: 1)$,

(10B, 2:0), (20B, 2:1), (20a, 2:1), (10D, 2:0), (20B, 2:1), (20b, 2:1), (10a, 3:2), (20A, 2:1),

$(10 A, 2: 0)$,

$(10 A, 2: 0)$,

$(4 a, 6: 5),(4 a, 6: 1),(10 C, 2: 0)$

$(20 a, 2: 1),(20 b, 2: 1)$,

(10B, 2:0), (10a, 3:2),

$(4 B, 5: 0),(10 b, 2: 0)$,

$(4 \widetilde{a}, 6: 5),(4 \widetilde{a}, 6: 1),(20 \widetilde{a}, 2: 1)$,

$(\widetilde{4}, 6: 5),(4 \widetilde{b}, 6: 1),(20 \widetilde{c}, 2: 1)$, $(20 \widetilde{c}, 2: 1)$,

$(4 \widetilde{a}, 5: 0)$,

$(10 B, 2: 1),(10 a, 3: 2)$,

$(4 \widetilde{b}, 5: 0)$,

$(3 A, 8: 7),(3 A, 8: 1),(21 A, 2: 1)$,

(7A, 3:0),

$(21 A, 2: 1)$

$(11 A, 3: 2),(11 A, 3: 1),(22 A, 2: 1)$, $(11 A, 2: 0)$,

$(12 A, 2: 0),(24 \widetilde{d}, 2: 1),(24 \widetilde{e}, 2: 1), \quad 24 A$

$(12 C, 2: 1)$, $(8 A, 4: 3),(8 A, 4: 1),(12 G, 2: 1)$, $(24 B, 2: 1),(24 \widetilde{j}, 2: 1)$, (12E, 2:0), (24A, 2:1), (24a, 2:1), $24 B$

$20 B$

$20 C$

$20 D$

$20 E$

$20 F$

$20 a$

$20 b$

$20 c$

$20 d$

$20 e$

$20 \widetilde{a}$

$20 \widetilde{b}$

$20 \widetilde{c}$

$20 \tilde{d}$

$20 \widetilde{e}$

$20 \tilde{f}$

$20 \widetilde{g}$

$20 \widetilde{h}$

$21 A$

$21 B$

$21 C$

$21 D$

$22 A$

$22 B$

$22 a$

$23 \mathrm{~A}$

$24 C$

Down

$(2: 1, \quad 20 D), \quad(2: 1, \quad 20 E)$, $(2: 0,40 A),(2: 0,40 b),(2: 0,40 c)$, $(2: 1,40 \widetilde{h}),(2: 1,40 \tilde{i}),(3: 0,60 c)$,

$(2: 0,40 d),(2: 1,40 \widetilde{k})$,

$(3: 0,60 F)$,

$(2: 0,40 C)$,

$(2: 1,20 D),(2: 1,20 c)$,

$(2: 1,20 E),(2: 1,20 c),(3: 0,60 e)$,

$(2: 1,40 \widetilde{k})$,

$(2: 0,40 e)$,

$(3: 2,20 A),(2: 1,20 \widetilde{b}),(2: 0,40 \widetilde{a})$,

$(4: 3,60 \widetilde{b}),(4: 1,60 \widetilde{b}),(3: 0,60 \widetilde{h})$,

$(3: 2, \quad 20 C), \quad(3: 0, \quad 60 \widetilde{f})$,

$(5: 4,100 \widetilde{b}),(5: 0,100 \widetilde{b})$,

$(2: 1,20 A),(2: 1,20 \widetilde{d}),(2: 1,20 \widetilde{e})$,

$(2: 0,40 \widetilde{b}),(2: 0,40 \widetilde{c}),(2: 0,40 \widetilde{d})$,

$(3: 0,60 \tilde{j})$,

$(2: 1,20 C),(2: 0,40 \widetilde{e})$,

$(2: 1,20 C),(2: 0,40 \widetilde{f}),(3: 0,60 \tilde{i})$,

$(2: 0,40 \widetilde{g}),(3: 2,100 \widetilde{a})$,

$(2: 0,40 \widetilde{k})$,

$(2: 0,40 \widetilde{j}),(3: 2,100 \widetilde{c})$,

$(2: 1,21 B),(2: 1,21 D),(2: 0,42 a)$,

$(2: 0,42 b),(3: 0,63 a)$,

$(3: 2,42 C),(3: 1,42 C),(2: 1,63 \widetilde{a})$, $(3: 1,84 C)$,

$(2: 0,42 d)$,

$(2: 1,22 B),(2: 0,44 a),(2: 0,44 c)$, $(2: 0,44 b)$,

$(3: 2,46 A),(3: 1,46 A),(3: 1,92 A)$, $(2: 1, \quad 24 D), \quad(2: 1, \quad 24 H)$, $(2: 0,48 a),(2: 0,48 b),(2: 1,48 \widetilde{j})$, $(2: 1,48 \widetilde{k}),(3: 0,72 b)$,

$(2: 1,24 C),(2: 1,24 I),(2: 0,48 A)$,

$24 D \quad(2: 1,48 \tilde{n})$, 
Up

$(8 B, 3: 0),(12 D, 2: 0),(24 \widetilde{l}, 2: 1)$, $(24 \widetilde{n}, 2: 1)$,

$(12 F, 2: 0),(24 d, 2: 1),(24 e, 2: 1)$, $(8 C, 4: 3),(8 C, 4: 1),(12 G, 2: 0)$, (24f, 2:1), (24g, 2:1),

(12H, 2:0), (24A, 2:1), (24b, 2:1), $(12 F, 2: 1),(24 B, 2: 1),(24 \widetilde{k}, 2: 1)$, $(8 F, 3: 0),(12 J, 2: 0),(24 i, 2: 1)$, $(24 j, 2: 1)$,

(12A, 2:0), (24 $\widetilde{b}, 3: 2)$,

(12A, 2:0), (24 $\widetilde{a}, 3: 2)$,

(8a, 4:3), (8a, 4:1), (12B, 2:0), $(24 a, 2: 1),(24 b, 2: 1),(24 \widetilde{c}, 3: 2)$, (12C, 2:0),

$(12 C, 2: 0)$,

$(12 C, 2: 0)$,

$(12 C, 2: 0)$,

(12E, 2:0), (12c, 2:1), (12d, 2:1), $(8 b, 3: 0),(12 e, 2: 0)$,

$(8 c, 3: 0),(12 e, 2: 0)$,

(12 $\widetilde{a}, 2: 0)$,

$(12 \widetilde{a}, 2: 0)$,

$(8 \widetilde{a}, 4: 3),(8 \widetilde{a}, 4: 1),(12 \widetilde{b}, 2: 0), \quad 24 \widetilde{c}$ $(24 \widetilde{a}, 2: 1),(24 \widetilde{b}, 2: 1)$,

$(12 \widetilde{d}, 2: 0)$,

$(12 \widetilde{d}, 2: 0)$,

$(12 \widetilde{d}, 2: 0)$, $(12 \widetilde{e}, 2: 0),(\underset{\widetilde{e}}{24}, 2: 1),(24 \widetilde{f}, 2: 1)$, $(8 \widetilde{b}, 4: 3),(8 \widetilde{b}, 4: 1),(12 \widetilde{f}, 2: 0)$, $(24 \widetilde{d}, 2: 1),(24 \widetilde{f}, 2: 1)$,

$(12 \widetilde{c}, 2: 0)$,

$(12 C, 2: 1)$,

$(12 C, 2: 1)$,

$(8 \widetilde{b}, 3: 0),(12 \widetilde{g}, 2: 0)$,

$(12 C, 2: 1)$,

$(8 \widetilde{c}, 3: 0),(12 \widetilde{g}, 2: 0)$,

f

$24 I$

$24 c$
Down

$24 E \quad(2: 0,48 g),(2: 1,72 \widetilde{r})$,

$24 F \quad(3: 0,72 e)$,

$24 G$

$24 H \quad(2: 0,48 h)$,

$24 \mathrm{~J}$

$24 a \quad(2: 1,24 D),(2: 1,24 c)$,

$24 b \quad(2: 1,24 H),(2: 1,24 c)$,

$24 d \quad(2: 1,24 F),(2: 1,48 A),(2: 0,48 e)$, $(2: 0,48 f),(2: 1,48 \widetilde{g}),(3: 0,72 c)$,

$24 e \quad(2: 1,24 F),(2: 1,48 \widetilde{h}),(2: 1,48 \widetilde{i})$, $(3: 0,72 d)$,

$24 f \quad(2: 1,24 G),(2: 1,48 \widetilde{g}),(2: 1,48 \widetilde{i})$, $24 g \quad(2: 1,24 G),(2: 1,48 A),(2: 0,48 c)$, $(2: 0,48 d),(2: 1,48 \widetilde{h})$,

$24 h \quad(2: 1,48 \widetilde{n})$,

$24 i \quad(2: 1,24 J),(2: 1,144 \widetilde{c})$,

$24 j \quad(2: 1,24 J),(2: 1,144 \widetilde{d})$,

$24 \widetilde{a} \quad(3: 2,24 b),(2: 1,24 \widetilde{c}),(3: 0,72 \widetilde{d})$,

$24 \widetilde{b} \quad(3: 2,24 a),(2: 1,24 \widetilde{c}),(3: 0,72 \widetilde{b})$, $(3: 0,72 \widetilde{e}),(5: 0,120 \widetilde{k})$,

$(3: 2,24 c),(3: 0,72 \widetilde{f})$,

$24 \widetilde{d} \quad(2: 1,24 A),(2: 1,24 \widetilde{h}),(2: 0,48 \widetilde{d})$, $(2: 0,48 \widetilde{e}),(3: 0,72 \widetilde{l}),(5: 0,120 \widetilde{n})$, $24 \widetilde{e} \quad(2: 1,24 A),(2: 1,24 \widetilde{g}),(2: 0,48 \widetilde{a})$, $(2: 0,48 \widetilde{b}),(3: 0,72 \widetilde{g}),(3: 0,72 \widetilde{m})$, $24 \widetilde{f} \quad(2: 1,24 \widetilde{g}),(2: 1,24 \widetilde{h}),(3: 0,72 \widetilde{h})$, $24 \widetilde{g} \quad(2: 0,48 \widetilde{f}),(3: 0,72 \widetilde{n})$,

$24 \widetilde{h} \quad(2: 0,48 \widetilde{c})$,

$2 \widetilde{4} \widetilde{i}(3: 2,24 \widetilde{s})$,

$2 \widetilde{j} \quad(2: 1,24 C),(2: 1,24 \widetilde{r}),(2: 0,48 \widetilde{g})$, $24 \widetilde{k} \quad(2: 1,24 I),(2: 1,24 \widetilde{o}),(2: 0,48 \widetilde{h})$, $24 \widetilde{l}(2: 1,24 E),(2: 0,48 \widetilde{o}),(2: 1,72 \widetilde{c})$, $24 \widetilde{m} \quad(2: 1,24 \widetilde{o}),(2: 1,24 \widetilde{r}),(2: 0,48 \widetilde{i})$, $24 \widetilde{n} \quad(2: 1,24 E),(2: 0,48 \widetilde{l}),(2: 0,48 \widetilde{m})$, $(2: 1,72 \widetilde{i}),(3: 0,72 \widetilde{s})$, 
Up

$(8 \widetilde{d}, 4: 3),(8 \widetilde{d}, 4: 1),(12 G, 2: 1), \quad 24 \widetilde{o}$ $(24 \widetilde{k}, 2: 1),(24 \widetilde{m}, 2: 1)$,

$(12 \widetilde{g}, 2: 0)$,

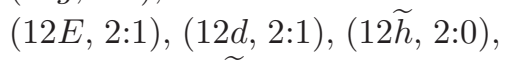

$24 \widetilde{p}$

$(12 F, 2: 1),(24 \widetilde{j}, 2: 1),(24 \widetilde{m}, 2: 1)$,

(12D, 2:0), (24 $\widetilde{i}, 3: 2)$,

$(12 e, 2: 0)$,

$(5 a, 3: 2)$,

$(5 B, 5: 4),(5 B, 5: 0),(25 A, 2: 1)$,

$(26 A, 2: 1)$,

$(13 A, 2: 0)$,

$(9 a, 3: 2),(9 b, 3: 2)$,

$(9 A, 3: 0)$,

$(9 A, 3: 0),(9 d, 2: 1)$,

$(9 B, 3: 0),(27 a, 2: 1)$,

$(9 b, 3: 0)$,

$(9 b, 3: 0)$,

(14A, 2:0),

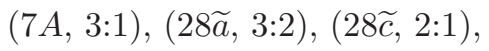
$(14 B, 2: 1),(14 B, 2: 0),(14 a, 3: 2)$, $(28 B, 2: 1)$,

$(14 C, 2: 0),(28 A, 2: 1),(28 a, 2: 1)$, (14A, 2:0),

$(4 \widetilde{a}, 8: 7),(4 \widetilde{a}, 8: 1),(28 \widetilde{a}, 2: 1)$,

$(28 \widetilde{c}, 2: 1)$

$(30 B, 2: 1)$,

$(15 A, \quad 3: 2), \quad(15 A, \quad 3: 1), \quad 30 C$ $(30 B, 2: 1)$, $(30 B, 2: 1)$,

(10D, 3:0), (30b, 2:1),

$(30 B, 2: 1)$,

$\begin{array}{llll}(15 C, & 3: 2), \quad(15 C, & 3: 1), \quad 30 G\end{array}$

$(30 A, \quad 2: 1), \quad(30 C, \quad 2: 1)$,

$(30 F, 2: 1)$,
$24 \widetilde{q}$

$24 \widetilde{r}$

$24 \widetilde{s}$

$24 \widetilde{t}$

$25 \mathrm{~A}$

$25 a$

$26 \mathrm{~A}$

$26 B$

$26 a$

$27 \mathrm{~A}$

$27 a$

$27 b$

$27 c$

$27 d$

$27 e$

$28 \mathrm{~A}$

$28 B$

$28 C$

$28 D$

$28 a$

$28 \widetilde{a}$

$28 \widetilde{b}$

$28 \widetilde{c}$

$28 \tilde{d}$

$29 \mathrm{~A}$

$30 A$

$30 B$

$30 D \quad(2: 0,60 E)$,

$30 E \quad(2: 0,60 F)$,

$30 F \quad(2: 1,30 G)$, $(2: 0,48 \widetilde{n})$,

$(2: 1,72 \widetilde{r})$,

$(2: 1,25 a)$, $(2: 0,52 B)$,

$(2: 1,27 c)$,

$(2: 0,54 c)$,

$(2: 0,54 d)$

$(2: 0,56 B)$,

$(2: 1,28 D)$, $(2: 0,56 \widetilde{b})$,

$(2: 0,56 \widetilde{f})$,

$(2: 0,58 a)$,

Down

$(2: 1,72 \widetilde{c}),(2: 1,72 \widetilde{i}),(3: 0,72 \widetilde{t})$,

$(2: 1,144 \widetilde{c}),(2: 1,144 \widetilde{d})$,

$(2: 1,26 B),(2: 0,52 A),(2: 0,52 a)$,

(2:1, 28D), (2:1, 56A), (2:0, 56b), $(2: 0,56 c),(2: 1,56 \widetilde{g})$,

$(2: 1,28 C),(2: 0,56 a),(3: 0,84 C)$,

$(3: 2,28 B),(2: 1,28 \widetilde{b}),(2: 0,56 \widetilde{a})$,

$(2: 0,56 \widetilde{c}),(3: 0,84 \widetilde{c}),(5: 0,140 \widetilde{e})$,

$(2: 1,28 B),(2: 1,28 \widetilde{d}),(2: 0,56 \widetilde{d})$, $(2: 0,56 \widetilde{e}),(3: 0,84 \widetilde{h})$,

$(2: 1,30 G),(3: 0,90 b)$,

$(2: 1, \quad 30 A), \quad(2: 1, \quad 30 C)$,

$(2: 1, \quad 30 D), \quad(2: 1, \quad 30 F)$,

$(2: 0,60 A),(2: 0,60 b),(3: 0,90 a)$,

$(2: 1,30 G),(2: 0,60 a),(2: 1,60 \widetilde{m})$, 
Up

$(15 A, 2: 0)$,

$(10 A, 3: 0)$,

$(10 a, 4: 3),(10 a, 4: 1),(15 B, 2: 0), \quad 30 c$ $(30 a, 2: 1),(30 d, 2: 1)$,

(15A, 2:0),

$(6 A, 5: 0)$,

$(6 b, 5: 0),(15 b, 2: 0)$,

(15a, 2:0),

$(16 A, 2: 1)$,

$(16 C, 2: 0),(16 a, 2: 1),(16 b, 2: 1)$,

$(16 a, 2: 0)$,

(16A, 2:0), (16g, 2:1), (16h, 2:1),

$(16 b, 2: 0)$,

$(16 b, 2: 0)$,

(16d, 2:0), (32c, 2:1), (32d, 2:1), $(16 \widetilde{b}, 2: 0)$,

$(16 \widetilde{c}, 2: 0)$,

$(16 e, 2: 1),(16 f, 2: 1),(16 \widetilde{d}, 2: 0)$, $(16 a, 2: 1),(16 c, 2: 1),(16 \widetilde{e}, 2: 0)$, $(16 A, 2: 1)$,

$(11 A, \quad 4: 3), \quad(11 A, \quad 4: 1)$, $(33 B, 2: 1)$,

$(17 A, 2: 0)$,

$(35 A, 2: 1)$, (7A, 5:0),

$(9 A, 3: 1),(12 D, 2: 1),(36 \widetilde{a}, 3: 2)$, $(36 \widetilde{d}, 2: 1),(36 \widetilde{e}, 2: 1)$,

$(9 B, 3: 1),(12 B, 3: 2),(12 B, 3: 0)$, $(36 A, 2: 1),(36 \widetilde{c}, 3: 2),(36 \widetilde{h}, 2: 1)$, $(36 \widetilde{k}, 2: 1)$,

$(12 e, 2: 1),(12 \widetilde{i}, 2: 1),(18 B, 2: 0)$, (18c, 3:2), (36A, 2:1), $(12 b, 3: 0),(18 a, 2: 0)$, $(9 a, 3: 1),(12 A, 3: 0),(36 \widetilde{b}, 3: 2)$,
$(36 \widetilde{n}, 2: 1)$,

$(12 b, 3: 0),(18 h, 2: 0)$, (12C, 3:0), (18a, 2:0), $(12 d, 3: 0),(18 e, 2: 0),(36 c, 2: 1), \quad 36 e$ $(36 h, 2: 1)$,
Down

$30 a$

$(2: 1,30 c),(3: 2,60 D),(3: 2,60 a)$, $(3: 2,60 \tilde{m})$,

$30 b \quad(2: 1,30 E),(2: 0,60 c),(2: 0,60 e)$, $(2: 1,90 \widetilde{a})$,

$30 d \quad(2: 1,30 c)$,

$30 e \quad(2: 0,60 d)$,

$30 f$

$30 \widetilde{a}$

$31 A \quad(3: 0,93 A)$,

$32 A$

$32 B$

$32 a \quad(3: 0,96 a)$,

$32 b \quad(2: 0,64 a)$,

$32 c \quad(2: 1,32 e)$,

$32 d \quad(2: 1,32 e)$,

$32 e$

$32 \widetilde{a}$

$32 \widetilde{b}$

$32 \widetilde{c}$

$32 \widetilde{d}$

$32 \widetilde{e}$

$33 A$

$33 B$

$34 A$

$(2: 1,33 A),(2: 0,66 a)$,

$(2: 0,68 A)$,

$34 a$

$35 A$

$35 B$

$35 a$

$36 A \quad(2: 1,36 B),(2: 1,36 D),(2: 0,72 a)$,

$36 B$

$(2: 1,35 B),(2: 0,70 a)$,

$36 C \quad(2: 1,72 \widetilde{p}),(2: 1,72 \widetilde{q})$,

$36 D$

$36 a \quad(2: 1,36 g)$,

$36 b \quad(2: 0,72 b)$

$36 c \quad(2: 1,36 e)$,

$36 d \quad(2: 1,36 g),(2: 0,72 c),(2: 0,72 d)$, 
Up f Down

(18C, 2:0), (18c, 3:2),

$(12 F, 3: 0),(18 d, 2: 0),(36 a, 2: 1), \quad 36 g$

$(36 d, 2: 1)$,

(12a, 3:0), (18h, 2:0),

$(12 f, 3: 0),(18 j, 2: 0)$,

$(12 \widetilde{c}, 2: 1)$,

$(12 \widetilde{a}, 3: 0)$,

$(12 \widetilde{b}, 3: 2),(12 \widetilde{b}, 3: 0),(36 \widetilde{a}, 2: 1)$, $(12 \widetilde{g}, 2: 1)$,

$(12 \widetilde{g}, 2: 1)$,

$(12 \widetilde{a}, 3: 0)$,

$(12 \widetilde{b}, 3: 0),(36 \widetilde{f}, 2: 1)$,

$(12 \widetilde{f}, 3: 2),(12 \widetilde{f}, 3: 0),(36 \widetilde{e}, 2: 1)$, (12 $\widetilde{d}, 3: 0)$,

$(12 \widetilde{e}, 3: 0),(36 \widetilde{i}, 2: 1)$,

$(36 \widetilde{e}, 2: 1)$,

(6 $\widetilde{a}, 3: 2),(12 D, 2: 1)$,

$(6 F, 2: 1),(6 \widetilde{a}, 3: 2)$,

$(12 \widetilde{d}, 3: 0)$,

$(12 f, 2: 1),(12 \widetilde{i}, 2: 1),(18 \widetilde{a}, 2: 0)$, $(12 \widetilde{c}, 3: 0)$,

(18C, 2:1), (18c, 3:2),

$(12 \widetilde{g}, 3: 0)$,

$(12 \widetilde{i}, 3: 0),(18 j, 2: 0)$,

$(19 A, 2: 0)$,

$(13 A, 3: 0)$,

$(39 A, 2: 1)$,

$(20 B, 2: 0)$,

$(20 A, 2: 0),(40 \widetilde{b}, 2: 1),(40 \widetilde{c}, 2: 1)$, (20F, 2:0), (40B, 2:1), (40a, 2:1), $(20 A, 2: 0),(40 \widetilde{a}, 3: 2)$,

$(20 B, 2: 0)$,

$(20 B, 2: 0)$,

$(20 D, 2: 0),(40 b, 2: 1),(40 c, 2: 1)$,

$40 a$
$36 f$

$36 h \quad(2: 1,36 e)$,

$36 i \quad(2: 1,108 \widetilde{k})$,

$36 \widetilde{a} \quad(3: 2,36 A),(2: 1,36 \widetilde{c}),(2: 0,72 \widetilde{a})$, $(3: 0,108 \widetilde{a}),(3: 0,108 \widetilde{c})$,

$36 \widetilde{b} \quad(3: 2,36 b),(2: 0,72 \widetilde{b}),(3: 2,108 \widetilde{b})$, $(5: 0,180 \widetilde{g})$,

$36 \widetilde{c} \quad(3: 2,36 B),(3: 0,108 \widetilde{d})$,

$36 \widetilde{d} \quad(2: 1,36 A),(2: 0,72 \widetilde{i}),(3: 0,108 \widetilde{f})$,

$36 \widetilde{e} \quad(2: 1,36 A),(2: 1,36 \widetilde{h}),(2: 1,36 \widetilde{k})$, $(2: 0,72 \widetilde{c}),(3: 0,108 \widetilde{i})$,

$36 \widetilde{f} \quad(2: 1,36 \widetilde{g}),(2: 0,72 \widetilde{d}),(2: 0,72 \widetilde{e})$, $(3: 2, \quad 108 \widetilde{b}), \quad(3: 0, \quad 108 \widetilde{e})$, $(3: 0,108 \widetilde{h})$,

$36 \widetilde{g} \quad(2: 0,72 \widetilde{f})$,

$36 \widetilde{h} \quad(2: 1,36 B)$,

$36 \tilde{i} \quad(2: 1,36 \tilde{j}),(2: 0,72 \widetilde{g}),(2: 0,72 \widetilde{h})$, $(3: 2,108 \widetilde{g})$,

$36 \widetilde{j} \quad(2: 0,72 \widetilde{n})$,

$36 \widetilde{k} \quad(2: 1,36 B),(3: 0,108 \tilde{j})$,

$36 \tilde{l} \quad(2: 0,72 \widetilde{r})$,

$36 \widetilde{m} \quad(2: 0,72 \widetilde{o})$,

$36 \widetilde{n} \quad(2: 1,36 b),(2: 0,72 \widetilde{l}),(2: 0,72 \widetilde{m})$,

$(3: 2,108 \widetilde{g})$,

$36 \widetilde{o} \quad(3: 0,108 \widetilde{k})$,

$36 \widetilde{p} \quad(2: 1,108 \widetilde{a})$,

$36 \widetilde{q}$

$36 \widetilde{r} \quad(2: 0,72 \widetilde{s}),(2: 0,72 \widetilde{t}),(2: 1,108 \widetilde{f})$,

$36 \widetilde{s} \quad(2: 1,108 \widetilde{k})$,

$38 A \quad(2: 0,76 a)$,

$38 a$

$39 A$

$39 B$

$39 C$

$40 \mathrm{~A}$

$40 B$

$40 C$

$40 b$

$40 c$

$(2: 1,39 C),(3: 0,117 a)$,

$40 d$
$(2: 1,80 \widetilde{d}),(2: 1,80 \widetilde{e})$,

$(2: 1,40 C),(2: 0,80 a)$,

$(2: 1,40 C)$,

$(2: 1,40 d),(2: 1,80 \widetilde{d})$,

$(2: 1,40 d),(2: 1,80 \widetilde{e})$, 
Up

(8C, 5:0), (20e, 2:0),

$(20 \widetilde{a}, 2: 0)$,

$(20 \widetilde{c}, 2: 0)$,

$(20 \widetilde{c}, 2: 0)$,

$(20 \widetilde{c}, 2: 0)$,

$(8 \widetilde{c}, 6: 5),(8 \widetilde{c}, 6: 1),(20 \widetilde{d}, 2: 0), \quad 40 \widetilde{e}$ $(40 \widetilde{b}, 2: 1),(40 \widetilde{d}, 2: 1)$,

$(20 \widetilde{e}, 2: 0),(40 \widetilde{c}, 2: 1),(40 \widetilde{d}, 2: 1)$, $(8 \widetilde{a}, 5: 0),(20 \widetilde{f}, 2: 0)$,

$(20 B, 2: 1)$,

$(20 B, 2: 1)$,

$(8 \widetilde{b}, 5: 0),(20 \widetilde{h}, 2: 0)$,

$(20 D, 2: 1),(20 d, 2: 1),(20 \widetilde{g}, 2: 0)$,

$(40 \widetilde{h}, 2: 1),(40 \widetilde{i}, 2: 1)$,

$(42 A, 2: 1)$,

$(14 B, \quad 3: 0), \quad(21 C, \quad 3: 2), \quad 42 C$

(21C, 3:1), (42c, 2:1),

$(42 A, 2: 1)$,

$(21 A, 2: 0)$,

$(21 A, 2: 0)$,

$(14 A, 3: 0)$,

(21D, 2:0), (42a, 2:1), (42b, 2:1),

(11A, 3:1), (44 $\widetilde{a}, 3: 2),(44 \widetilde{b}, 2: 1)$,

$(22 A, 2: 0)$,

(22B, 2:0), (44a, 2:1), (44c, 2:1),

(22A, 2:0),

(15a, 2:1),

$(15 A, 3: 0)$,

$(15 C, 3: 0),(45 a, 2: 1)$,

$(9 a, 5: 0),(15 b, 3: 0)$,

$(23 A, \quad 3: 2), \quad(23 A, \quad 3: 1), \quad 46 A$ $(46 C, 2: 1)$,

$(24 B, 2: 0),(24 d, 2: 1),(24 g, 2: 1)$,
$42 D$

$40 e$

$40 \widetilde{a}$

$40 \widetilde{b}$

$(3: 2, \quad 40 a), \quad(4: 3, \quad 120 \widetilde{b})$, $(4: 1,120 \widetilde{b})$,

Down

$(2: 1,40 B),(2: 1,40 \widetilde{e}),(2: 0,80 \widetilde{b})$, $(2: 0,80 \widetilde{c})$,

$40 \widetilde{c} \quad(2: 1,40 B),(2: 1,40 \widetilde{f}),(2: 0,80 \widetilde{a})$, $(3: 0,120 \widetilde{l})$,

$40 \widetilde{d} \quad(2: 1, \quad 40 \widetilde{e}), \quad(2: 1, \quad 40 \widetilde{f})$, $(3: 0,120 \widetilde{m})$,

$40 \widetilde{f} \quad(3: 0,120 \tilde{j})$,

$40 \widetilde{g}$

$40 \widetilde{h} \quad(2: 1,40 \widetilde{k}),(2: 0,80 \widetilde{d})$,

$40 \widetilde{i} \quad(2: 1,40 \widetilde{k}),(2: 0,80 \widetilde{e})$,

$40 \tilde{j} \quad(2: 0,80 \widetilde{f})$,

$40 \widetilde{k}$

$41 A \quad(2: 0,82 a)$

$42 A \quad(2: 1, \quad 42 B), \quad(2: 1, \quad 42 D)$,

$(2: 0,84 A),(2: 0,84 a)$,

$42 B \quad(2: 0,84 B)$,

$42 a$

$42 b$

$42 c$

$42 d$

$44 \mathrm{~A}$

$44 a$

$44 b$

$44 c$

$44 \widetilde{a}$

$(2: 1,44 b)$,

$(3: 2, \quad 44 A), \quad(2: 0, \quad 88 \widetilde{a})$, $(4: 3,132 \widetilde{a}),(4: 1,132 \widetilde{a})$,

$44 \widetilde{b} \quad(2: 1,44 A),(2: 0,88 \widetilde{b}),(2: 0,88 \widetilde{c})$,

$45 \mathrm{~A}$

$45 a$

$45 b$

$45 c$

$$
(2: 1,45 b)
$$

$46 C \quad(2: 1,46 A)$, 
Up

$(24 A, 2: 0)$,

(24A, 2:0),

$(24 g, 2: 0)$,

$(24 g, 2: 0)$,

(24d, 2:0),

(24d, 2:0),

$(16 a, 3: 0),(24 E, 2: 0),(48 \widetilde{l}, 2: 1)$,

$(48 \widetilde{m}, 2: 1)$,

$(24 H, 2: 0),(48 a, 2: 1),(48 b, 2: 1)$,

(24) $, 2: 0)$,

(24 $\widetilde{e}, 2: 0)$,

$(16 \widetilde{a}, 4: 3),(16 \widetilde{a}, 4: 1),(24 \widetilde{h}, 2: 0)$,

$(48 \widetilde{d}, 2: 1),(48 \widetilde{e}, 2: 1)$,

$(24 \tilde{d}, 2: 0)$,

$(24 \widetilde{d}, 2: 0)$,

$(24 \widetilde{g}, 2: 0),(48 \widetilde{a}, 2: 1),(48 \widetilde{b}, 2: 1)$,

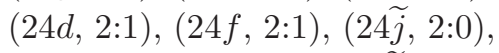

(24e, $2: 1),(24 g, 2: 1),(24 \widetilde{k}, 2: 0)$,

$(24 e, 2: 1),(24 f, 2: 1),(24 \widetilde{m}, 2: 0)$,

(24A, 2:1),

(24A, 2:1),

$(16 \widetilde{b}, 3: 0),(24 \widetilde{n}, 2: 0)$,

(16 $\widetilde{c}, 3: 0),(24 \widetilde{n}, 2: 0)$,

$(24 \underset{\sim}{ }, 2: 1),(24 h, 2: 1),(24 \widetilde{q}, 2: 0), \quad 48 \widetilde{n}$

$(48 \widetilde{j}, 2: 1),(48 \widetilde{k}, 2: 1)$,

$(24 \widetilde{l}, 2: 0)$,

(1A, 28:21),

(10b, 3:2),

(50A, 2:1),

$(26 A, 2: 0)$,

$(26 B, 2: 0),(52 A, 2: 1),(52 a, 2: 1)$, (26A, 2:0),

$(4 \widetilde{a}, 14: 13),(4 \widetilde{a}, 14: 1),(52 \widetilde{a}, 2: 1), \quad 52 \widetilde{b}$

$(52 \widetilde{c}, 2: 1)$,

(18a, 3:2), (18h, 3:2),

$(18 B, 3: 0)$,

(18E, 3:0), (54a, 2:1),

(18c, 3:0), (27b, 2:0),
$48 h$

$48 \widetilde{a}$

$48 \widetilde{b}$

$48 \widetilde{c}$

$48 \tilde{d}$

$48 \widetilde{e}$

$48 \tilde{f}$

$48 \widetilde{g}$

$48 \widetilde{h}$

$48 \tilde{i}$

$48 \tilde{j}$

$48 \widetilde{k}$

$48 \tilde{l}$

$48 \tilde{m}$

$48 \widetilde{o}$

$49 a$

$50 A$

$50 a$

$51 \mathrm{~A}$

$52 A$

$52 B$

$52 a$

$52 \widetilde{a}$

$52 \widetilde{c}$

$52 \widetilde{d}$

$54 \mathrm{~A}$

$54 a$

$54 b$

$54 c$ $\mathrm{f}$

Down
$(2: 1,48 \widetilde{f}),(3: 0,144 \widetilde{a})$,

$(2: 1,48 \widetilde{f}),(3: 0,144 \widetilde{b})$,

$(2: 1,48 \widetilde{c})$,

$(2: 1,48 \widetilde{c})$,

$(3: 0,144 \widetilde{e})$,

$(2: 0,96 \widetilde{a})$,

$(2: 0,96 \widetilde{b})$,

$(2: 1,48 \widetilde{n})$,

$(2: 1,48 \widetilde{n})$,

(2:1, 48g),

$(2: 1,48 g)$,

$(2: 1,50 a)$

(2:0, 102a),

$(2: 1,52 B),(2: 0,104 A)$,

$(2: 1,52 B)$,

$(2: 1, \quad 52 \widetilde{b}), \quad(2: 0, \quad 104 \widetilde{a})$, $(3: 0,156 \widetilde{b})$,

$(2: 1, \quad 52 \widetilde{d}), \quad(2: 0, \quad 104 \widetilde{b})$, $(2: 0,104 \widetilde{c})$, $(2: 0,104 \widetilde{d})$

$(2: 1,54 b)$, 
(18g, 3:0), (27d, 2:0),

$(18 j, 2: 1),(18 \widetilde{a}, 3: 0)$,

$(28 A, 2: 1)$,

(28D, 2:0), (56b, 2:1), (56c, 2:1),

$(28 B, 2: 0),(56 \widetilde{a}, 3: 2)$,

(28A, 2:0),

$(28 A, 2: 0)$,

$(28 \widetilde{a}, 2: 0)$,

$(8 \widetilde{a}, 8: 7),(8 \widetilde{a}, 8: 1),(28 \widetilde{b}, 2: 0), \quad 56 \widetilde{b}$

$(56 \widetilde{a}, 2: 1),(56 \widetilde{c}, 2: 1)$,

$(28 \widetilde{a}, 2: 0)$,

$(28 \widetilde{c}, 2: 0)$,

$(28 \widetilde{c}, 2: 0)$,

$(28 \widetilde{d}, 2: 0),(56 \widetilde{d}, 2: 1),(56 \widetilde{e}, 2: 1)$, $(28 A, 2: 1)$,

$(19 A, 3: 0)$,

$(29 A, 2: 0)$,

$(30 B, 2: 0)$,

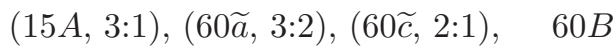

$(15 C, 3: 1),(60 B, 2: 1),(60 \widetilde{d}, 3: 2), \quad 60 C$ $(60 \widetilde{e}, 2: 1),(60 \widetilde{l}, 2: 1)$,

(30a, 3:2), (60B, 2:1),

(30D, 2:0), (60A, 2:1), (60b, 2:1),

$(20 E, 3: 0),(30 E, 2: 0),(60 c, 2: 1)$,

(60e, 2:1),

(30C, 2:0), (30a, 3:2),

$(30 B, 2: 0)$,

$(20 B, 3: 0),(30 b, 2: 0)$,

$(12 a, 5: 0),(30 e, 2: 0)$,

$(20 b, 3: 0),(30 b, 2: 0)$,

$(20 \widetilde{a}, 4: 3),(20 \widetilde{a}, 4: 1),(60 \widetilde{a}, 2: 1)$,

$(60 \widetilde{a}, 2: 1)$

$(60 \widetilde{c}, 2: 1)$,

$(12 \widetilde{c}, 6: 5),(12 \widetilde{c}, 6: 1),(20 \widetilde{b}, 3: 0)$,

$(60 \widetilde{h}, 2: 1)$,
$60 D$

$54 d$

$54 \widetilde{a}$

$56 A$

$56 B$

$56 a$

$56 b \quad(2: 1,56 B)$,

$56 c \quad(2: 1,56 B)$,

$56 \widetilde{a} \quad(3: 2,56 a),(2: 1,56 \widetilde{b})$,

$56 \widetilde{c} \quad(2: 1,56 \widetilde{b})$,

$56 \widetilde{d} \quad(2: 1,56 \widetilde{f})$,

$56 \widetilde{e} \quad(2: 1, \quad 56 \widetilde{f}), \quad(2: 0, \quad 112 \widetilde{a})$, $(2: 0,112 \widetilde{b})$,

$56 \tilde{f} \quad(2: 0,112 \widetilde{c})$,

$56 \widetilde{g}$

$57 \mathrm{~A}$

$58 a$

$60 \mathrm{~A}$

$(2: 1, \quad 60 E), \quad(2: 1, \quad 120 \widetilde{g})$,

$(2: 1,120 \widetilde{h})$,

$(2: 1, \quad 60 C), \quad(2: 1, \quad 60 D)$,

$(2: 0,120 a)$,

$60 E$

$60 F$

$60 a$

$60 b \quad(2: 1,60 E)$,

$60 c \quad(2: 1,60 F)$,

$60 d$

$60 e \quad(2: 1,60 F)$,

$60 \widetilde{a} \quad(3: 2,60 B),(2: 1,60 \widetilde{b}),(2: 1,60 \widetilde{d})$,

$(2: 0, \quad 120 \widetilde{a}), \quad(2: 0, \quad 120 \widetilde{c})$,

$(3: 0,180 \widetilde{b})$,

$60 \widetilde{b} \quad(2: 0,120 \widetilde{b})$,

$60 \widetilde{c} \quad(2: 1,60 B),(2: 1,60 \widetilde{e}),(2: 1,60 \widetilde{g})$,

$(2: 1, \quad 60 \widetilde{l}), \quad(2: 0, \quad 120 \widetilde{d})$,

$(2: 0,120 \widetilde{f}),(3: 0,180 \widetilde{d})$,

$60 \widetilde{d} \quad(3: 2,60 C),(3: 0,180 \widetilde{c})$,

$60 \widetilde{e} \quad(2: 1,60 C),(3: 0,180 \widetilde{e})$, 
Up f Down

$(60 \widetilde{c}, 2: 1)$,

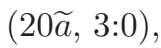

$(20 \widetilde{e}, 3: 0),(60 \tilde{j}, 2: 1)$,

$(20 \widetilde{c}, 3: 0)$,

$(12 \widetilde{a}, 5: 0)$,

$(60 \widetilde{c}, 2: 1)$,

$(30 C, 2: 1),(30 a, 3: 2)$,

$(12 \widetilde{d}, 5: 0)$,

(21A, 3:0),

$(21 C, 2: 1)$,

(32b, 2:0),

$(66 A, 2: 1)$,

$(33 B, 2: 0)$,

(34A, 2:0),

(70A, 2:1),

(35A, 2:0),

(36A, 2:0), (72 $\widetilde{a}, 3: 2)$,

$(24 A, 3: 0),(36 b, 2: 0),(72 \widetilde{l}, 2: 1)$,

$(72 \widetilde{m}, 2: 1)$,

$(24 d, 3: 0),(36 d, 2: 0)$,

(24e, 3:0), (36d, 2:0),

$(24 F, 3: 0),(36 g, 2: 0),(72 c, 2: 1)$,

(72d, 2:1),

(36 $\widetilde{a}, 2: 0)$,

$(24 \widetilde{b}, 3: 0),(36 \widetilde{b}, 2: 0)$,

$(24 \widetilde{l}, 2: 1),(24 \widetilde{p}, 2: 1),(36 \widetilde{e}, 2: 0)$,

$(24 \widetilde{a}, 3: 0),(36 \widetilde{f}, 2: 0)$,

$(24 \widetilde{b}, 3: 0),(36 \widetilde{f}, 2: 0)$,

$(24 \widetilde{c}, 3: 0),(36 \widetilde{g}, 2: 0),(72 \widetilde{d}, 2: 1), \quad 72 \widetilde{f}$

(72 $, 2: 1)$,

$(24 \widetilde{e}, 3: 0),(36 \widetilde{i}, 2: 0)$,

$(24 \widetilde{f}, 3: 0),(36 \tilde{i}, 2: 0)$,

$(24 \widetilde{n}, 2: 1),(24 \widetilde{p}, 2: 1),(36 \widetilde{d}, 2: 0)$,

(12e, 2:1),

(12e, 2:1),

$(24 \widetilde{d}, 3: 0),(36 \widetilde{n}, 2: 0)$,

$63 \widetilde{a}$

$66 \mathrm{~A}$

$66 a$

$68 \mathrm{~A}$

$70 a$

$72 a$

$72 b$

$72 c$

$72 e$

$72 \widetilde{a}$

$72 \widetilde{b}$

$72 \widetilde{c}$
$60 \widetilde{g} \quad(2: 0,120 \widetilde{i})$,

$60 \widetilde{h} \quad(2: 1, \quad 60 \widetilde{f}), \quad(2: 0, \quad 120 \widetilde{e})$,

$(2: 1,180 \widetilde{a})$,

$60 \widetilde{i} \quad(2: 0,120 \tilde{j})$,

$60 \tilde{j} \quad(2: 1, \quad 60 \tilde{i}), \quad(2: 0, \quad 120 \widetilde{l})$,

$(2: 0,120 \widetilde{m}),(2: 1,180 \widetilde{f})$,

$60 \widetilde{k} \quad(2: 0,120 \widetilde{k}),(3: 0,180 \widetilde{g})$,

$60 \tilde{l} \quad(2: 1,60 C)$,

$60 \widetilde{m}$

$60 \widetilde{n} \quad(2: 0,120 \widetilde{n})$,

$63 a \quad(2: 0,126 a)$,

$64 a$

$66 B$

$(2: 1,66 B),(2: 0,132 a)$,

$68 \widetilde{a} \quad(2: 0,136 \widetilde{a})$,

$68 \widetilde{b} \quad(2: 0,136 \widetilde{b})$,

$70 A \quad(2: 1,70 B),(2: 0,140 a)$,

$70 B$

$(2: 1,72 e)$,

$72 d \quad(2: 1,72 e)$,

$72 \widetilde{d} \quad(2: 1,72 \widetilde{f})$,

$72 \widetilde{e} \quad(2: 1,72 \widetilde{f}),(3: 0,216 \widetilde{b})$,

$72 \widetilde{g} \quad(2: 1, \quad 72 \widetilde{n}), \quad(2: 0, \quad 144 \widetilde{a})$, $(2: 0,144 \widetilde{b})$,

$72 \widetilde{h} \quad(2: 1,72 \widetilde{n})$,

$72 \widetilde{i} \quad(3: 0,216 \widetilde{c})$,

$72 \widetilde{j} \quad(2: 1,72 \widetilde{o}),(2: 0,144 \widetilde{c})$,

$72 \widetilde{k} \quad(2: 1,72 \widetilde{o}),(2: 0,144 \widetilde{d})$,

$72 \widetilde{l} \quad(2: 1,72 b)$, 
$\mathrm{Up}$

$(24 \widetilde{e}, 3: 0),(36 \widetilde{n}, 2: 0)$,

$(24 \widetilde{g}, 3: 0),(36 \widetilde{j}, 2: 0),(72 \widetilde{g}, 2: 1), \quad 72 \widetilde{n}$

$(72 \widetilde{h}, 2: 1)$,

$(12 J, 2: 1),(12 \widetilde{j}, 2: 1),(36 \widetilde{m}, 2: 0), \quad 72 \widetilde{o}$

$(72 \widetilde{j}, 2: 1),(72 \widetilde{k}, 2: 1)$,

$(36 C, 2: 1)$,

$(36 C, 2: 1)$,

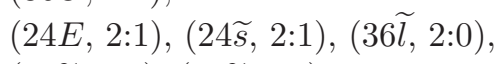

$(24 \widetilde{n}, 3: 0),(36 \widetilde{r}, 2: 0)$,

$(24 \widetilde{p}, 3: 0),(36 \widetilde{r}, 2: 0)$,

$(38 A, 2: 0)$,

$(78 A, 2: 1)$,

$(40 B, 2: 0),(80 \widetilde{b}, 2: 1),(80 \widetilde{c}, 2: 1)$,

$(40 \widetilde{c}, 2: 0)$,

$(40 \widetilde{b}, 2: 0)$,

$(40 \tilde{b}, 2: 0)$,

(40A, 2:1), (40b, 2:1), (40 $\widetilde{h}, 2: 0)$,

(40A, 2:1), (40c, 2:1), (40 $\widetilde{i}, 2: 0)$,

$(16 \widetilde{a}, 5: 0),(40 \widetilde{j}, 2: 0)$,

(41A, 2:0),

$(42 A, 2: 0)$,

(42B, 2:0), (84A, 2:1), (84a, 2:1),

$(21 C, 3: 1),(28 B, 3: 0),(84 \widetilde{c}, 3: 2)$

$(84 \widetilde{h}, 2: 1)$,

(42A, 2:0),

$(12 \widetilde{a}, 8: 7),(12 \widetilde{a}, 8: 1),(84 \widetilde{a}, 2: 1)$, $(28 \widetilde{a}, 3: 0)$,

$(84 \widetilde{a}, 2: 1)$,

$(84 \widetilde{e}, 2: 1)$

$(84 \widetilde{e}, 2: 1)$,

$(28 \widetilde{c}, 3: 0)$,

$(44 A, 2: 0),(88 \widetilde{b}, 2: 1),(88 \widetilde{c}, 2: 1)$,

$(44 \widetilde{a}, 2: 0)$,

$(44 \widetilde{b}, 2: 0)$,

$(44 \widetilde{b}, 2: 0)$,

$(30 B, 3: 0)$,

f

$72 \widetilde{p}$

$72 \widetilde{q}$

$72 \widetilde{r}$

$72 \widetilde{s}$

$72 \widetilde{t}$

$76 a$

$76 \widetilde{a}$

$76 \tilde{b}$

$78 A$

$78 B$

$80 a$

$80 \widetilde{a}$

$80 \widetilde{b}$

$80 \widetilde{c}$

$80 \widetilde{d}$

$80 \widetilde{e}$

$80 \tilde{f}$

$82 a$

$84 A$

$84 B$

$84 C$

$84 a$

$84 \widetilde{b}$

$84 \widetilde{g}$

$84 \widetilde{h}$

$88 \mathrm{~A}$

$88 \widetilde{a}$

$88 \widetilde{b}$
Down

$(2: 1,72 b)$,

$(2: 0,144 \widetilde{e})$,

$(2: 1,84 B)$,

$84 \widetilde{a} \quad(2: 1,84 \widetilde{b}),(2: 1,84 \widetilde{d}),(2: 0,168 \widetilde{a})$,

$(2: 0,168 \widetilde{b}),(3: 0,252 \widetilde{a})$,

$84 \widetilde{c} \quad(3: 2,84 C),(2: 1,252 \widetilde{b})$,

$84 \widetilde{d} \quad(2: 0,168 \widetilde{e})$,

$84 \widetilde{e} \quad(2: 1,84 \widetilde{f}),(2: 1,84 \widetilde{g}),(2: 0,168 \widetilde{c})$,

$(2: 0,168 \widetilde{d})$,

$84 \widetilde{f} \quad(2: 0,168 \widetilde{f})$,

$(2: 1,84 C),(2: 1,252 \widetilde{c})$,

$(2: 1,88 A)$,

$88 \widetilde{c} \quad(2: 1,88 A)$,

$90 a \quad(2: 1,90 b)$, 
Up

(30A, 3:0), (90a, 2:1),

(30b, 2:1), f

Down

$90 b$

$90 \widetilde{a}$

(23A, 3:1), (92 $\widetilde{a}, 3: 2),(92 \widetilde{b}, 2: 1), \quad 92 A$

$92 \widetilde{a} \quad(3: 2,92 A)$,

$92 \widetilde{b}(2: 1,92 A)$,

(31A, 3:0),

$93 A$

$(32 a, 3: 0),(48 g, 2: 0)$,

$96 a$

$(48 e, 2: 1),(48 f, 2: 1),(48 \widetilde{g}, 2: 0), \quad 96 \widetilde{a}$

$(48 c, 2: 1),(48 d, 2: 1),(48 \widetilde{h}, 2: 0), \quad 96 \widetilde{b}$

$(20 \tilde{f}, 3: 2)$,

$100 \widetilde{a}(2: 1,100 \widetilde{b})$,

$(20 \widetilde{b}, 5: 4),(20 \widetilde{b}, 5: 0),(100 \widetilde{a}, 2: 1), \quad 100 \widetilde{b}$

$(20 \widetilde{h}, 3: 2)$,

$(100 \widetilde{c}, 2: 1)$,

$100 \widetilde{c}(2: 1,100 \widetilde{d})$,

(51A, 2:0),

$100 \widetilde{d}$

$(52 A, 2: 0)$,

$102 a$

$(52 \widetilde{a}, 2: 0)$,

$104 \mathrm{~A}$

$(52 \widetilde{c}, 2: 0)$,

$104 \widetilde{a}$

$(52 \widetilde{c}, 2: 0)$,

$(52 \widetilde{d}, \quad 2: 0), \quad(104 \widetilde{b}, \quad 2: 1), \quad 104 \widetilde{d}$

$104 \widetilde{b} \quad(2: 1,104 \widetilde{d})$,

$104 \widetilde{c}(2: 1,104 \widetilde{d}),(2: 0,208 \widetilde{a})$,

$(104 \widetilde{c}, 2: 1)$,

$(36 \widetilde{a}, 3: 0),(36 \widetilde{p}, 2: 1)$,

$(36 \widetilde{b}, 3: 2),(36 \widetilde{f}, 3: 2)$,

$108 \widetilde{a} \quad(2: 0,216 \widetilde{a})$,

$(36 \widetilde{a}, 3: 0)$,

$108 \widetilde{b}$

$(36 \widetilde{c}, 3: 0),(108 \widetilde{c}, 2: 1)$,

$108 \widetilde{c}$

$108 \widetilde{d}$

$(36 \tilde{f}, 3: 0)$,

$108 \widetilde{e} \quad(2: 0,216 \widetilde{b})$,

$(36 \widetilde{d}, 3: 0),(36 \widetilde{r}, 2: 1)$,

$108 \widetilde{f}(2: 0,216 \widetilde{c})$,

$(36 \tilde{i}, 3: 2),(36 \widetilde{n}, 3: 2)$,

$108 \widetilde{g}$

$(36 \widetilde{f}, 3: 0)$,

$108 \widetilde{h}$

(36 $\widetilde{e}, 3: 0)$,

$108 \tilde{i}$

$(2: 1,108 \tilde{j})$

$(36 \widetilde{k}, 3: 0),(108 \widetilde{i}, 2: 1)$,

$108 \tilde{j}$

$(36 i, 2: 1),(36 \widetilde{o}, 3: 0),(36 \widetilde{s}, 2: 1), \quad 108 \widetilde{k}$

$(54 \widetilde{a}, 2: 0)$,

(56 $\widetilde{e}, 2: 0)$,

$112 \widetilde{a} \quad(2: 1,112 \widetilde{c})$,

$(56 \widetilde{e}, 2: 0)$,

$112 \widetilde{b}(2: 1,112 \widetilde{c})$,

$(56 \widetilde{f}, \quad 2: 0), \quad(112 \widetilde{a}, \quad 2: 1), \quad 112 \widetilde{c}$

$(112 \widetilde{b}, 2: 1)$,

$(39 A, 3: 0)$,

$116 \widetilde{a} \quad(2: 0,232 \widetilde{a})$,

$117 a$

$(60 B, 2: 0),(120 \widetilde{a}, 3: 2)$,

$120 a$

(60व $, 2: 0)$,

$120 \widetilde{a}(3: 2,120 a),(2: 1,120 \widetilde{b})$,

$(40 \widetilde{a}, 4: 3),(40 \widetilde{a}, 4: 1),(60 \widetilde{b}, 2: 0), \quad 120 \widetilde{b}$

$(120 \widetilde{a}, 2: 1),(120 \widetilde{c}, 2: 1)$,

$(60 \widetilde{a}, 2: 0)$,

$120 \widetilde{c}(2: 1,120 \widetilde{b})$, 
Up f Down

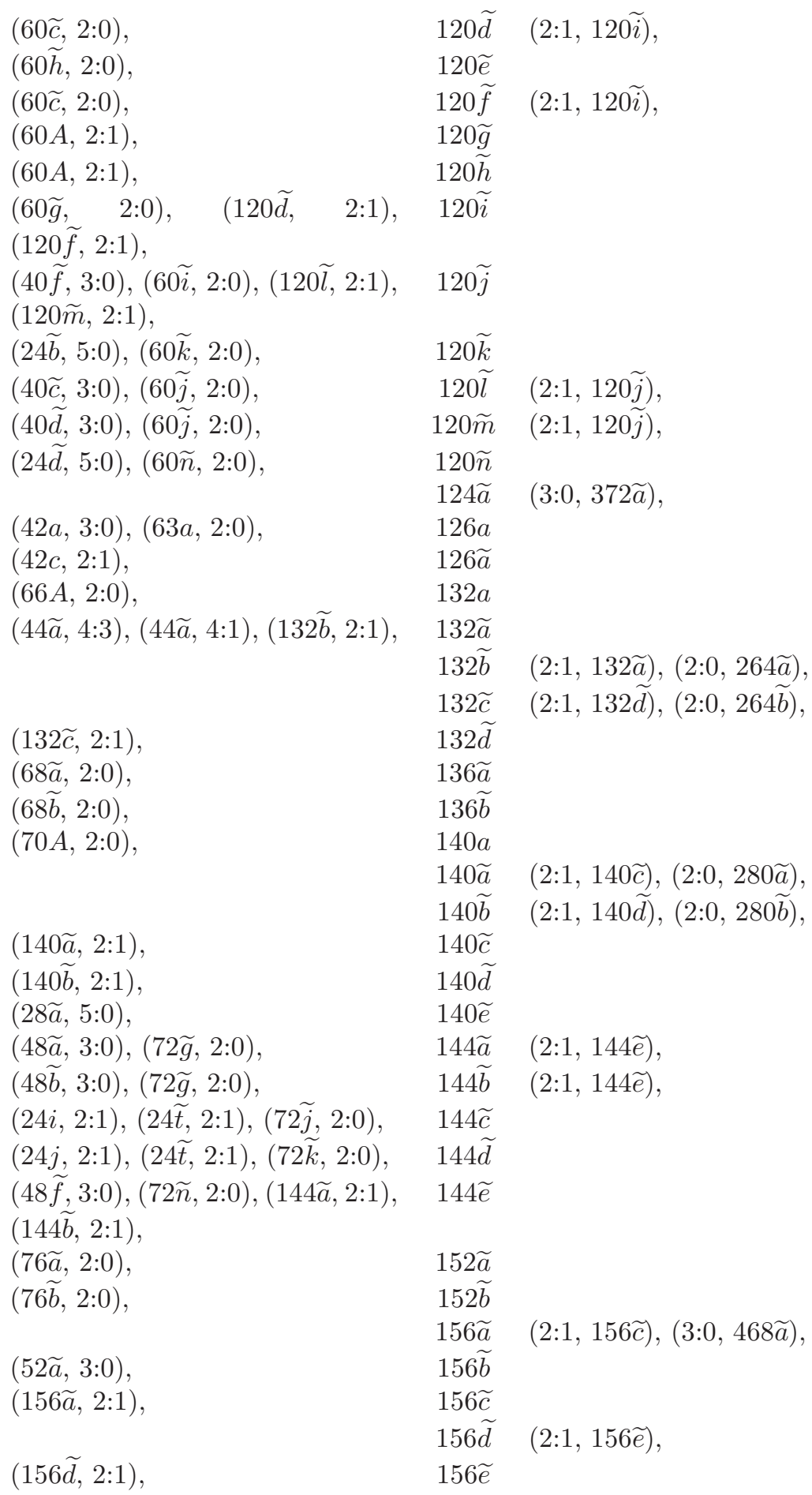


Up $\quad$ f Down

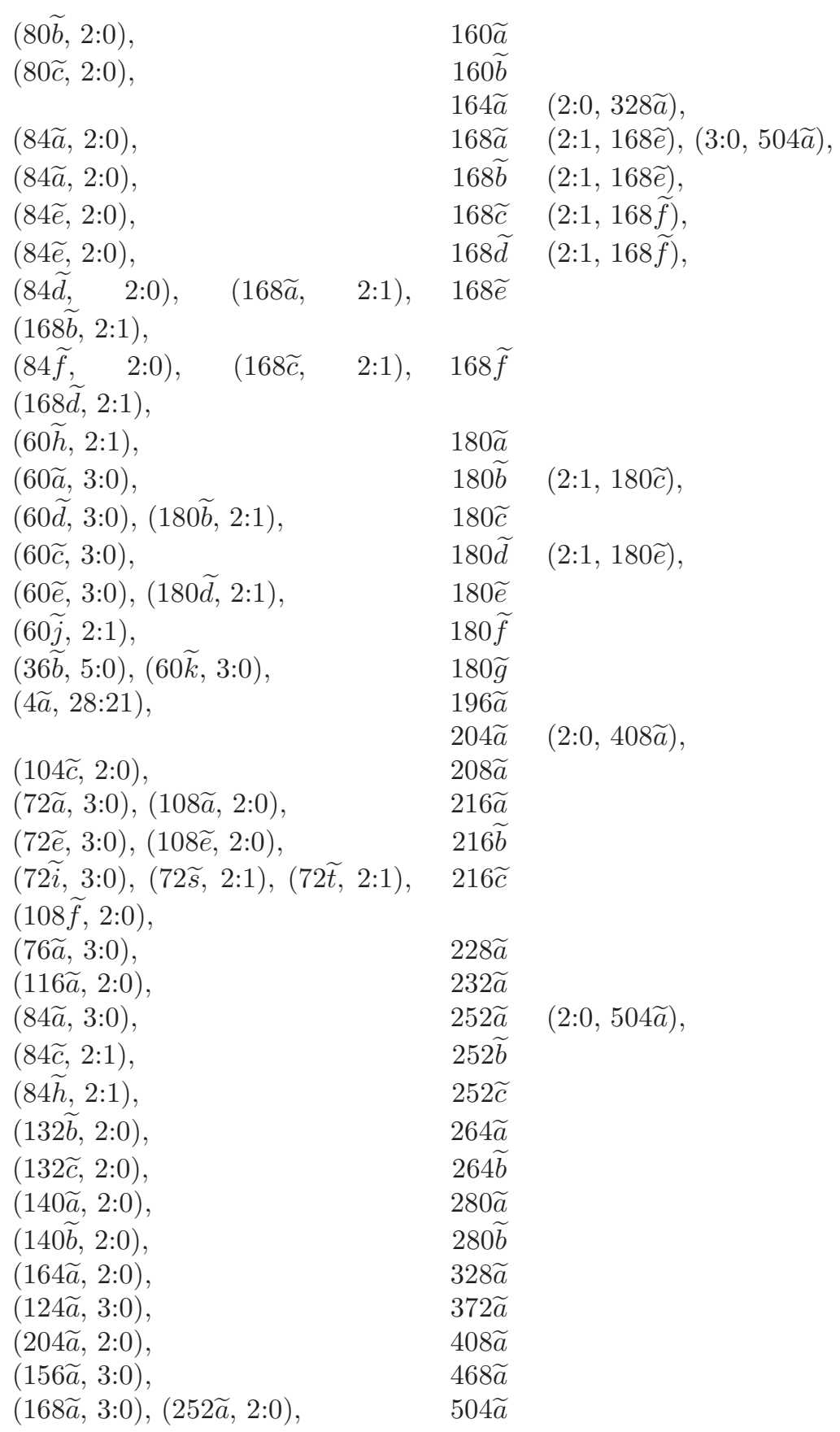

The connected components of the graph are:

One with 480: $\{1 A, 2 A, 2 B, 2 a, 3 A, 3 B, 3 C, 4 A, 4 B, 4 C, 4 D, 4 a, 4 \widetilde{a}, 4 \widetilde{b}, 5 A, 5 B$, $5 a, 6 A, 6 B, 6 C, 6 D, 6 E, 6 F, 6 a, 6 b, 6 c, 6 d, 6 \widetilde{a}, 7 A, 7 B, 8 A, 8 B, 8 C, 8 D, 8 E, 8 F$, 
$8 a, 8 b, 8 c, 8 \widetilde{a}, 8 \widetilde{b}, 8 \widetilde{c}, 8 \widetilde{d}, 9 A, 9 B, 9 a, 9 b, 9 c, 9 d, 10 A, 10 B, 10 C, 10 D, 10 E, 10 a$, $10 b, 10 c, 12 A, 12 B, 12 C, 12 D, 12 E, 12 F, 12 G, 12 H, 12 I, 12 J, 12 a, 12 b, 12 c, 12 d$, $12 e, 12 f, 12 \widetilde{a}, 12 \widetilde{b}, 12 \widetilde{c}, 12 \widetilde{d}, 12 \widetilde{e}, 12 \widetilde{f}, 12 \widetilde{g}, 12 \widetilde{h}, 12 \widetilde{i}, 12 \widetilde{j}, 13 A, 13 B, 14 A, 14 B$, $14 C, 14 a, 14 b, 14 c, 15 A, 15 B, 15 C, 15 D, 15 a, 15 b, 16 A, 16 B, 16 C, 16 a, 16 b, 16 c$, $16 d, 16 e, 16 f, 16 g, 16 h, 16 \widetilde{a}, 16 \widetilde{b}, 16 \widetilde{c}, 16 \widetilde{d}, 16 \widetilde{e}, 18 A, 18 B, 18 C, 18 D, 18 E, 18 a$, $18 b, 18 c, 18 d, 18 e, 18 f, 18 g, 18 h, 18 i, 18 j, 18 \widetilde{a}, \underset{\sim}{2} A, 20 B, 20 C, 20 D, 20 E, 20 F$, $20 a, 20 b, 20 c, 20 d, 20 e, 20 \widetilde{a}, 20 \widetilde{b}, 20 \widetilde{c}, 20 \widetilde{d}, 20 \widetilde{e}, 20 \widetilde{f}, 20 \widetilde{g}, 20 \widetilde{h}, 21 A, 21 B, 21 C, 21 D$, $24 A, 24 B, 24 C, 24 D, 24 E, 24 F, 24 G, 24 H, 24 I, 24 J, 24 a, 24 b, 24 c, 24 d, 24 e, 24 f$, $24 g, 24 h, 24 i, 24 j, 24 \widetilde{a}, 24 \widetilde{b}, 24 \widetilde{c}, 24 \widetilde{d}, 24 \widetilde{e}, 24 \widetilde{f}, 24 \widetilde{g}, 24 \widetilde{h}, 24 \widetilde{i}, 24 \widetilde{j}, 24 \widetilde{k}, 24 \widetilde{l}, 24 \widetilde{m}$, $24 \widetilde{n}, 24 \widetilde{o}, 24 \widetilde{p}, 24 \widetilde{q}, 24 \widetilde{r}, 24 \widetilde{s}, 24 \widetilde{t}, 25 A, 25 a, 26 a, 27 A, 27 a, 27 b, 27 c, 27 d, 27 e, 28 A$, $28 B, 28 C, 28 D, 28 a, 28 \widetilde{a}, 28 \widetilde{b}, 28 \widetilde{c}, 28 \widetilde{d}, 30 A, 30 B, 30 C, 30 D, 30 E, 30 F, 30 G, 30 a$, $30 b, 30 c, 30 d, 30 e, 30 f, 30 \widetilde{a}, 32 A, 32 B, 32 a, 32 b, 32 c, 32 d, 32 e, 32 \widetilde{a}, 32 \widetilde{b}, 32 \widetilde{c}, 32 \widetilde{d}$, $32 \widetilde{e}, 35 a, 36 A, 36 B, 36 C, 36 D_{2} 36 a, 36 b, 36 c, 36 d, 36 e, 36 f, 36 g, 36 h, 36 i, 36 \widetilde{a}$, $36 \widetilde{b}, 36 \widetilde{c}, 36 \widetilde{d}, 36 \widetilde{e}, 36 \widetilde{f}, 36 \widetilde{g}, 36 \widetilde{h}, 36 \tilde{i}, 36 \tilde{j}, 36 \widetilde{k}, 36 \widetilde{l}, 36 \widetilde{m}, 36 \widetilde{n}, 36 \widetilde{o}, 36 \widetilde{p}, 36 \widetilde{q}, 36 \widetilde{r}$, $36 \widetilde{s}, 39 B, 40 A, 40 B, 40 C, 40 a, 40 b, 40 c, 40 d, 40 e, 40 \widetilde{a}, 40 \widetilde{b}, 40 \widetilde{c}, 40 \tilde{d}, 40 \widetilde{e}, 40 \widetilde{f}$, $40 \widetilde{g}, 40 \widetilde{h}, 40 \widetilde{i}, 40 \widetilde{j}, 40 \widetilde{k}, 42 C, 42 a, 42 b, 42 c, 42 d, 45 A, 45 a, 45 b, 45 c, 48 \underset{\sim}{\sim}, 48 a, 48 b$, $48 c, 48 d, 48 e, 48 f, 48 g, 48 h, 48 \widetilde{a}, 48 \widetilde{b}, 48 \widetilde{c}, 48 \tilde{d}, 48 \widetilde{e}, 48 \widetilde{f}, 48 \widetilde{g}, 48 \widetilde{h}, 48 \widetilde{i}, 48 \widetilde{j}, 48 \widetilde{k}$, $48 \widetilde{l}, 48 \widetilde{m}, 48 \widetilde{n}, 48 \widetilde{o}, 49 a, 50 A, 50 a, 52 \widetilde{a}, 52 \widetilde{b}, 54 A, 54 a, 54 b, 54 c, 54 d, 54 \widetilde{a}, 56 A$, $56 B, 56 a, 56 b, 56 c, 56 \widetilde{a}, 56 \widetilde{b}, 56 \widetilde{c}, 56 \widetilde{d}, 56 \widetilde{e}, 56 \widetilde{f}, 56 \widetilde{g}, 60 A, 60 B, 60 C, 60 D, 60 E$, $60 \underset{\sim}{F}, 60 a, 60 b, 60 c, 60 d, 60 e, 60 \widetilde{a}, 60 \widetilde{b}, 60 \widetilde{c}, 60 \widetilde{d}, 60 \widetilde{e}, 60 \widetilde{f}, 60 \widetilde{g}, 60 \widetilde{h}, 60 \widetilde{i}, 60 \widetilde{j}, 60 \widetilde{k}$, $60 \widetilde{l}, 60 \widetilde{m}, 60 \widetilde{\sim}, 63 a, 63 \widetilde{a}, 64 a, 72 a, 72 b, 72 c, 72 d, 72 e, 72 \widetilde{a}, 72 b, 72 \widetilde{c}, 72 \widetilde{d}, 72 \widetilde{e}, 72 \widetilde{f}$, $72 \widetilde{g}, 72 \widetilde{h}, 72 \widetilde{i}, 72 \widetilde{j}, 72 \widetilde{k}, 72 \widetilde{l}, 72 \widetilde{m}, 72 \widetilde{n}, 72 \widetilde{o}, 72 \widetilde{p}, 72 \widetilde{q}, 72 \widetilde{r}, 72 \widetilde{s}, 72 \widetilde{t}, 80 a, 80 \widetilde{a}, 80 \widetilde{b}$, $80 \widetilde{c}, 80 \widetilde{d}, 80 \widetilde{e}, 80 \tilde{f}, 84 C, 84 \widetilde{a}, 84 \widetilde{b}, 84 \widetilde{c}, 84 \widetilde{d}, 84 \widetilde{h}, 90 a, 90 b, 90 \widetilde{a}, 96 a, 96 \widetilde{a}$, $96 \widetilde{b}, 100 \widetilde{a}, 100 \vec{b}, 100 \widetilde{c}, 100 \widetilde{d}, 104 \widetilde{a}, 108 \widetilde{a}, 108 \widetilde{b}, 108 \widetilde{c}, 108 \widetilde{d}, 108 \widetilde{e}, 108 \widetilde{f}, 108 \widetilde{g}, 108 \widetilde{h}$, $108 \widetilde{i}, 108 \widetilde{j}, 108 \widetilde{k}, 112 \widetilde{a}, 112 \widetilde{b}, 112 \widetilde{c}, 120 a, 120 \widetilde{a}, 120 \widetilde{b}, 120 \widetilde{c}, 120 \widetilde{d}, 120 \widetilde{e}, 120 \widetilde{f}, 120 \widetilde{g}$, $120 \widetilde{h}, 120 \tilde{i}, 120 \widetilde{j}, 120 \widetilde{k}, 120 \widetilde{\sim}, 120 \widetilde{m}, 120 \widetilde{n}, 126 a, 126 \widetilde{a}, 140 \widetilde{e}, 144 \widetilde{a}, 144 \widetilde{b}, 144 \widetilde{c}$, $144 \widetilde{d}, 144 \widetilde{e}, 156 \widetilde{b}, 160 \widetilde{a}, 160 \widetilde{b}, 168 \widetilde{a}, 168 \widetilde{b}, 168 \widetilde{e}, 180 \widetilde{a}, 180 \widetilde{b}, 180 \widetilde{c}, 180 \widetilde{d}, 180 \widetilde{e}, 180 \widetilde{f}$ $180 \widetilde{g}, 196 \widetilde{a}, 216 \widetilde{a}, 216 \widetilde{b}, 216 \widetilde{c}, 252 \widetilde{a}, 252 \widetilde{b}, 252 \widetilde{c}, 504 \widetilde{a}\}$.

One with 20: $\{11 A, 22 A, 22 B, 22 a, 33 A, 33 B, 44 A, 44 a, 44 b, 44 c, 44 \widetilde{a}, 44 \widetilde{b}, 66 a$, $88 A, 88 \widetilde{a}, 88 \widetilde{b}, 88 \widetilde{c}, 132 \widetilde{a}, 132 \widetilde{b}, 264 \widetilde{a}\}$.

Five with 6: $\{23 A, 46 A, 46 C, 92 A, 92 \widetilde{a}, 92 \widetilde{b}\},\{26 A, 26 B, 52 A, 52 B, 52 a, 104 A\}$, $\{42 A, 42 B, 42 D, 84 A, 84 B, 84 a\},\{52 \widetilde{c}, 52 \widetilde{d}, 104 \widetilde{b}, 104 \widetilde{c}, 104 \widetilde{d}, 208 \widetilde{a}\},\{84 \widetilde{e}, 84 \widetilde{f}$, $84 \widetilde{g}, 168 \widetilde{c}, 168 \widetilde{d}, 168 \widetilde{f}\}$.

Ten with 3: $\{19 A, 38 a, 57 A\},\{35 A, 35 B, 70 a\},\{39 A, 39 C, 117 a\},\{66 A, 66 B$, $132 a\},\{70 A, 70 B, 140 a\},\{76 \widetilde{a}, 152 \widetilde{a}, 228 \widetilde{a}\},\{132 \widetilde{c}, 132 \widetilde{d}, 264 \widetilde{b}\},\{140 \widetilde{a}, 140 \widetilde{c}, 280 \widetilde{a}\}$, $\{140 \widetilde{b}, 140 \widetilde{d}, 280 \widetilde{b}\},\{156 \widetilde{a}, 156 \widetilde{c}, 468 \widetilde{a}\}$.

Sixteen with 2: $\{17 A, 34 a\},\{29 A, 58 a\},\{31 A, 93 A\},\{34 A, 68 A\},\{38 A, 76 a\}$, $\{41 A, 82 a\},\{51 A, 102 a\},\{68 \widetilde{a}, 136 \widetilde{a}\},\{68 \widetilde{b}, 136 \widetilde{b}\},\{76 \widetilde{b}, 152 \widetilde{b}\},\{78 A, 78 B\},\{116 \widetilde{a}$, $232 \widetilde{a}\},\{124 \widetilde{a}, 372 \widetilde{a}\},\{156 \widetilde{d}, 156 \widetilde{e}\},\{164 \widetilde{a}, 328 \widetilde{a}\},\{204 \widetilde{a}, 408 \widetilde{a}\}$.

24 isolated: $47 \underset{\widetilde{\sim}}{\mathrm{f}}, 55 \mathrm{~A}, 59 A, 62 A, 69 A, 71 A, 87 A, 94 A, 95 A, 105 A, 110 A, 119 A$, $124 \widetilde{b}, 188 \widetilde{a}, 188 \widetilde{b}, 220 \widetilde{a}, 220 \widetilde{b}, 236 \widetilde{a}, 276 \widetilde{a}, 284 \widetilde{a}, 348 \widetilde{a}, 380 \widetilde{a}, 420 \widetilde{a}, 476 \widetilde{a}$.

\section{Acknowledgement}

We thank Simon P. Norton for his help and insight. 


\section{References}

1. D. Alexander, C. J. Cummins, J. MCKay and C. Simons, 'Completely replicable functions', Groups, combinatorics $\&$ geometry (Durham, 1990), London Math. Soc. Lecture Note Ser. 165 (Cambridge University Press, Cambridge, 1992) 87-98. 146

2. J. H. Conway, J. MCKAy and A. SEBbar, 'On the discrete groups of moonshine', Proc. Amer. Math. Soc. 132 (2004) 2233-2240. 146

3. J. H. Conway and S. P. Norton, 'Monstrous moonshine', Bull. London Math. Soc. 11 (1979) 308-339. 146

4. C. J. Cummins, 'Some comments on replicable functions', Modern trends in Lie algebra representation theory (Queen's Univ., Kingston, ON, 1994), Queen's Papers in Pure and Appl. Math. 94 (1994) 48-55. 146

5. D. Ford, J. MCKAY and S. P. Norton, 'More on replicable functions', Comm. in Algebra 22 (1994) 5175-5193. 146

6. J. Gutierrez, R. Rubio and D. Sevilla, 'Unirational fields of transcendence degree one and functional decomposition', Proceedings of International Symposium on Symbolic and Algebraic Computation, ISSAC 2001, 167-175. 147

7. J. MCKAY, 'Essentials of monstrous moonshine', Groups and combinatorics - in memory of Michio Suzuki, Adv. Stud. Pure Math. 32 (2001) 347-353. 146

8. S. P. Norton, 'More on moonshine', Computational group theory (London Academic Press, 1984) 185-193. 146

9. J. Gutierrez and D. Sevilla, 'Building counterexamples to generalizations for rational functions of Ritt's decomposition Theorem', Journal of Algebra 303 (2006) 655-667. 151

10. D. Sevilla, 'Teoremas de Ritt y computación de cuerpos unirracionales', PhD Thesis, University of Cantabria, 2004. 147

J. MCKay mckay@cs.concordia.ca

Department of Computer Science

Concordia University

Montreal H3G 1M8, QC, Canada

David Sevilla david.sevilla@ricam.oeaw.ac.at

Johann Radon Institute for Computational and Applied Mathematics (RICAM)

Altenbergerstrasse 69

A-4040 Linz, Austria 